\title{
Product kernels adapted to curves in the space
}

\section{Valentina Casarino, Paolo Ciatti and Silvia Secco}

\begin{abstract}
We establish $L^{p}$-boundedness for a class of operators that are given by convolution with product kernels adapted to curves in the space. The $L^{p}$ bounds follow from the decomposition of the adapted kernel into a sum of two kernels with singularities concentrated respectively on a coordinate plane and along the curve.

The proof of the $L^{p}$-estimates for the two corresponding operators involves Fourier analysis techniques and some algebraic tools, namely the Bernstein-Sato polynomials.

As an application, we show that these bounds can be exploited in the study of $L^{p}-L^{q}$ estimates for analytic families of fractional operators along curves in the space.
\end{abstract}

\section{Introduction}

The purpose of this paper is to establish $L^{p}$ boundedness for a class of product-type convolution operators.

In the last thirty years the theory of singular integrals on product domains has been largely developed. The first case which was considered is that of a convolution operator $T f=K * f$ on $\mathbb{R}^{d_{1}} \times \mathbb{R}^{d_{2}}$, with $K(x, y)=$ $K_{1}(x) K_{2}(y), x \in \mathbb{R}^{d_{1}}, y \in \mathbb{R}^{d_{2}}, K_{1}$ and $K_{2}$ being of Calderón-Zygmund type. In this case a simple iteration argument yields the $L^{p}$ boundedness of $T$. A more involved situation is that of a convolution operator $T$, whose kernel $K$ is defined on $\mathbb{R}^{d_{1}} \times \mathbb{R}^{d_{2}}$ and satisfies all the analogous bounds to those satisfied by $K_{1} K_{2}$ on $\mathbb{R}^{d_{1}} \times \mathbb{R}^{d_{2}}$, but cannot be decomposed as product of two kernels $K_{1}(x)$ and $K_{2}(y)$. A precise definition of such kernels, which are called "product kernels", was introduced in terms of certain differential inequalities and suitable cancellation conditions.

2000 Mathematics Subject Classification: 42B20, 44 A35.

Keywords: Product kernels, $L^{p}$ estimates, convolution, Bernstein-Sato polynomials. 
Several conditions on $K$, guaranteeing the $L^{p}$ boundedness of the operator $T$, have been introduced [4], and many applications of the product theory to the operators arising in certain boundary value problems have been studied [12], [11]. Moreover, the euclidean spaces $\mathbb{R}^{d_{j}}, j=1,2$, have been replaced by appropriate nilpotent groups [9], [12], and by smooth manifolds with a geometry determined by a control distance [10].

Recently, one of the authors studied the $L^{p}$ boundedness for convolution operators with product kernels adapted to curves in the plane [14], that is product-type kernels in $\mathbb{R}^{2}$ whose singularities are supported along a coordinate axis and a transversal curve. Here we extend these results to higher dimensional spaces. In order not to burden the exposition with notational complexities, we are going to give the full details only for $\mathbb{R}^{3}=\mathbb{R}^{d_{1}} \times \mathbb{R}^{d_{2}}$, with $d_{1}=1$ and $d_{2}=2$, proving $L^{p}$-bounds for convolution operators by kernels singular along a coordinate plane and a transversal curve of polynomial type. In the last section we shall quickly describe how the arguments should be modified in the higher dimensional setting.

We denote an element of $\mathbb{R}^{3}=\mathbb{R} \times \mathbb{R}^{2}$ by the pair $\left(x_{1}, x\right)$, where $x=$ $\left(x_{2}, x_{3}\right) \in \mathbb{R}^{2}$. On $\mathbb{R}$ we consider the usual dilations by $\delta>0$, while on $\mathbb{R}^{2}$ we consider the anisotropic dilation given by

$$
\delta \circ x=\left(\delta^{\frac{1}{2 n}} x_{2}, \delta^{\frac{1}{2 m}} x_{3}\right), \quad \text { with } \quad \delta>0, m, n \in \mathbb{N}, m<n .
$$

We denote by

$$
Q=\frac{1}{2 n}+\frac{1}{2 m}
$$

the homogeneous dimension of $\mathbb{R}^{2}$ with respect to the dilations (1.1) and by $\rho(x)=x_{2}^{2 n}+x_{3}^{2 m}$ a smooth homogeneous norm on $\mathbb{R}^{2}$.

In this context the proto-typical example of a product kernel in $\mathbb{R}^{3}$ (we refer to Section 3 for a precise definition) is given by the distribution

$$
H\left(x_{1}, x\right)=C_{\mu} p \cdot v \cdot \frac{1}{x_{1}} \rho(x)^{-Q+i \mu}, \quad \mu \in \mathbb{R} \backslash\{0\} .
$$

Throughout the paper we concentrate our attention on the convolution by product-type kernels in $\mathbb{R}^{3}$ whose singularities are supported on a coordinate plane and on a transversal curve of finite type. A rather simple example of such a kernel, obtained by adapting $H$ to the curve $\gamma: \mathbb{R} \rightarrow \mathbb{R}^{2}$, $\gamma\left(x_{1}\right)=\left(x_{1}^{m}, x_{1}^{n}\right)$, is given by

$$
K\left(x_{1}, x\right)=C_{\mu} p . v \cdot\left(\frac{1}{x_{1}}\right) \rho\left(x-\gamma\left(x_{1}\right)\right)^{-Q+i \mu}, \mu \in \mathbb{R} \backslash\{0\} .
$$

Notice that, if $m=n$, the subspace generated by $t \mapsto\left(t, t^{m}, t^{n}\right)$ is only two-dimensional, a situation which in principle is intermediate between the totally flat case and the one discussed in this paper. 
More generally, we introduce the following class of product-type kernels.

Definition 1.1. Assume that $K_{0}$ is a product kernel on $\mathbb{R}^{3}$ and consider the curve $x=\gamma\left(x_{1}\right)$ with $\gamma\left(x_{1}\right)=\left(x_{1}^{m}, x_{1}^{n}\right), x_{1} \in \mathbb{R}, m, n \in \mathbb{N}, 2 \leq m<n$. We define a distribution $K$ by

$$
\int K\left(x_{1}, x\right) f\left(x_{1}, x\right) d x_{1} d x:=\int K_{0}\left(x_{1}, x\right) f\left(x_{1}, x+\gamma\left(x_{1}\right)\right) d x_{1} d x
$$

for a Schwartz function $f$ on $\mathbb{R}^{3}$. $K$ will be called an adapted kernel.

Here with an abuse of notation we write pairings between distributions and test functions as integrals. The kernel $K$ given by the formula (1.4) is a well-defined tempered distribution which is singular on the coordinate plane $x_{1}=0$ and along the curve $x=\gamma\left(x_{1}\right), x_{1} \in \mathbb{R}$.

We shall prove the following theorem.

Theorem 1.2. Let $K$ be the distribution defined by the formula (1.4). Then the convolution operator $T: f \mapsto f * K$, initially defined on the Schwartz space $\mathcal{S}\left(\mathbb{R}^{3}\right)$, extends to a bounded operator on $L^{p}\left(\mathbb{R}^{3}\right)$ for $1<p<\infty$.

To prove Theorem 1.2 we decompose the adapted kernel $K$ of $T$ into the sum of a kernel $K_{1}$ with singularities concentrated on the coordinate plane $x_{1}=0$ and of a kernel $K_{2}$ singular along the curve $x=\gamma\left(x_{1}\right), x_{1} \in \mathbb{R}$. As in [14] we show that the multiplier associated with $K_{1}$ satisfies some Marcinkiewicz-type conditions, while $K_{2}$ is treated by means of analytic interpolation (our proof is inspired by some arguments used in [16] to prove the $L^{p}$ boundedness of the Hilbert transform along curves in the plane). In particular, to apply the analytic interpolation method we need to introduce a non-isotropic version of the Riesz potentials

$$
\mathcal{I}^{z}\left(u_{1}, u_{2}\right):=\left(\rho\left(u_{1}, u_{2}\right)\right)^{z-Q}, \quad z \in \mathbb{C} .
$$

and to determine their meromorphic continuation. To extend in a meromorphic way $\mathcal{I}^{z}$, we study the location of its singularities by using Bernstein-Sato polynomials. Since the reader is not assumed to be familiar with BernsteinSato functional identities, we illustrate the definition and the basic properties of this algebraic tool in Section 2.

The results illustrated in this paper find applications in several contexts in harmonic and complex analysis (for more on this, see [12]). In particular, in the last section we show that $L^{p}$ bounds for convolution by product kernels adapted to curves can be exploited in the study of $L^{p}-L^{q}$ estimates for analytic families of fractional operators [2]. 
Throughout the paper we will use the variable constant convention, and denote by $C$, possibly with sub- or superscripts, a constant that may vary from place to place.

It is a pleasure to thank Professor Fulvio Ricci for many helpful discussions on the subject of this paper.

\section{Bernstein-Sato polynomials and a family of Riesz- type kernels}

Consider the polynomial

$$
\rho\left(u_{1}, u_{2}\right):=u_{1}^{2 n}+u_{2}^{2 m},
$$

with $m, n \in \mathbb{N}, m \geq 1, n>m$. Observe that $\rho$ is homogeneous with respect to the one-parameter family of non isotropic dilations given by (1.1).

We shall often use, in the following, the relations between $\rho$ and the euclidean norm $|\cdot|$ in $\mathbb{R}^{2}$

$$
A \rho(u)^{\frac{1}{2 n}} \leq|u| \leq B \rho(u)^{\frac{1}{2 m}} \quad \text { if } \rho(u)>1
$$

and

$$
A^{\prime} \rho(u)^{\frac{1}{2 m}} \leq|u| \leq B^{\prime} \rho(u)^{\frac{1}{2 n}} \quad \text { if } \rho(u) \leq 1
$$

for some $A, B, A^{\prime}, B^{\prime}>0$.

Define now the distribution

$$
\mathcal{I}^{z}\left(u_{1}, u_{2}\right):=\left(\rho\left(u_{1}, u_{2}\right)\right)^{z-Q},
$$

where $z \in \mathbb{C}, \Re e z>0$.

Observe that both $\rho$ and $\mathcal{I}^{z}$ depend on $m$ and $n$. Anyway, for the sake of simplicity, we shall avoid to indicate the dependence on $m$ and $n$.

We collect in the next proposition some obvious properties of $\mathcal{I}^{z}$.

Proposition 2.1. If $\Re e z>0$, then

i) $\mathcal{I}^{z}$ is well defined as distribution and locally integrable.

ii) $\mathcal{I}^{z}$ is a tempered distribution.

iii) $\mathcal{I}^{z}$ is an analytic family of tempered distributions, that is, given $f \in$ $\mathcal{S}\left(\mathbb{R}^{2}\right)$, the functions $z \mapsto<\mathcal{I}^{z}, f>$ are holomorphic. 
We shall now prove that $\mathcal{I}^{z}$ admits a meromorphic extension, with poles in a at most countable set, consisting of rational negative points. Our method is based on the theory of Bernstein-Sato polynomials.

It is well-known in algebra that, given a non-zero polynomial $p\left(u_{1}, u_{2}\right)$ with complex coefficients, there exist a non-zero polynomial $b_{p}(s) \in \mathbb{C}[s]$ and a differential operator $L(s)$ whose coefficients are polynomials in $s, u_{1}, u_{2}$, such that formally

$$
L(s)\left(p\left(u_{1}, u_{2}\right)\right)^{s+1}=b_{p}(s)\left(p\left(u_{1}, u_{2}\right)\right)^{s} \text { for all } s \in \mathbb{C} .
$$

The set of all polynomials $b_{p}(s) \in \mathbb{C}[s]$, such that there exists an operator $L$ for which this formal identity is satisfied, forms an ideal, and the unique monic generator of this ideal is called the Bernstein-Sato polynomial of $p$.

In our case, for $\Re e z>0$ we may write

$$
\left(\rho\left(u_{1}, u_{2}\right)\right)^{z-Q}=\frac{L(z-Q)\left(\rho\left(u_{1}, u_{2}\right)\right)^{z+1-Q}}{b_{\rho}(z-Q)} .
$$

By repeatedly using the functional equation (2.6) we may extend $\mathcal{I}^{z}$ to the complex plane in a meromorphic way, with poles whenever $b_{\rho}(z-Q+k)$ vanishes for a non-negative integer $k$. Therefore we shall now seek for the zeros of the Bernstein-Sato polynomial $b_{\rho}(z-Q)$.

According to a theorem of Kashiwara, the roots of the Bernstein-Sato polynomial are negative rational numbers. Moreover, if $\rho$ has the particularly simple form given in (2.1), it is easy to find the roots of $b_{\rho}(s)$.

Lemma 2.2. If $b_{\rho}(s)$ denotes the Bernstein-Sato polynomial associated to $\rho\left(u_{1}, u_{2}\right):=u_{1}^{2 n}+u_{2}^{2 m}, m<n, m, n \in \mathbb{N}$, then the roots of $\frac{b_{\rho}(s)}{s+1}$ are given by

$$
-\frac{p_{1}}{2 n}-\frac{p_{2}}{2 m}, \quad 1 \leq p_{1} \leq 2 n-1, \quad 1 \leq p_{2} \leq 2 m-1,
$$

with multiplicity one.

Proof. It is essentially due to Kashiwara [7]. See also [8] and [1, Remark 3.8].

In the following corollary we collect some observations, which will be useful in the following.

Corollary 2.3. $i)$ The largest root of $b_{\rho}(s)$ is $-Q=-\frac{1}{2 m}-\frac{1}{2 n}$.

ii) -1 is a root of $b_{\rho}(s)$ with multiplicity two.

iii) The set of the roots of $b_{\rho}(s)$ is symmetric with respect to -1 . 
Proof. $i$ ) It follows obviously from (2.7) for $p_{1}=1$ and $p_{2}=1$.

ii) Observe that $-\frac{p_{1}}{2 n}-\frac{p_{2}}{2 m}=-1$ for $p_{1}=n$ and $p_{2}=m$. Then -1 is a root of multiplicity one for $\frac{b_{\rho}(s)}{s+1}$, whence the thesis follows.

iii) Suppose that the $-1+\delta:=-\frac{\bar{p}_{1}}{2 n}-\frac{\bar{p}_{2}}{2 m}$ is a root of $b_{\rho}(s)$ for some $\bar{p}_{1}, \bar{p}_{2} \in \mathbb{N}$, $1 \leq \bar{p}_{1} \leq 2 n-1,1 \leq \bar{p}_{2} \leq 2 m-1$ and some $\delta>0$. Take $\tilde{p}_{1}:=2 n-\bar{p}_{1}$ and $\tilde{p}_{2}:=2 m-\bar{p}_{2}$. Since $1 \leq \tilde{p}_{1} \leq 2 n-1,1 \leq \tilde{p}_{2} \leq 2 m-1$, then

$$
-\frac{\tilde{p}_{1}}{2 n}-\frac{\tilde{p}_{2}}{2 m}=-2+\frac{\bar{p}_{1}}{2 n}+\frac{\bar{p}_{2}}{2 m}=-1-\delta
$$

is a root of $b_{\rho}(s)$.

Example 2.4. By means of formula (2.7) it is possible to find the roots of the Bernstein-Sato polynomial associated to $u_{1}^{6}+u_{2}^{4}$ and one finds

$$
\begin{aligned}
b_{\rho}(s)= & (s+1)^{2}\left(s+\frac{2}{3}\right)\left(s+\frac{4}{3}\right)\left(s+\frac{3}{4}\right)\left(s+\frac{5}{4}\right)\left(s+\frac{5}{6}\right)\left(s+\frac{7}{6}\right) \\
& \left(s+\frac{5}{12}\right)\left(s+\frac{19}{12}\right)\left(s+\frac{7}{12}\right)\left(s+\frac{17}{12}\right)\left(s+\frac{11}{12}\right)\left(s+\frac{13}{12}\right) .
\end{aligned}
$$

Let us now consider the distribution $\mathcal{I}^{z}$ defined by $(2.4)$. Let $s_{1}, \ldots, s_{h}$ be the zeros of $b_{\rho}(s)$ in $(-Q-1,-Q]$, each counted with its multiplicity and ordered in a decreasing way. Then the meromorphic continuation of $\mathcal{I}^{z}$ has poles whenever $z=Q+s_{j}-k, k \geq 0$. We remark, in particular, that 0 and $-1+Q$ are always poles for $\mathcal{I}^{z}$. By gluing all together, we get the following result.

Proposition 2.5. $\mathcal{I}^{z}$ may be analytically continued to a meromorphic distribution-valued function of $z$, also denoted by $\mathcal{I}^{z}$, with poles in a set $A$, consisting of rational negative points. More precisely,

$$
A=\left\{\zeta_{j, k}:=Q+s_{j}-k: k \in \mathbb{N}, j=1, \ldots, h\right\},
$$

$s_{j}, j=1, \ldots, h$, denoting the zeros of the Bernstein-Sato polynomial $b_{\rho}$ in $(-Q-1,-Q]$, each listed as many times as its multiplicity. Each pole has order one, with the exception of the points $-1+Q-k, k \in \mathbb{N}$, which have order two.

Set $\zeta_{j}:=\zeta_{j, 0}$. Observe that $\zeta_{1}=0$ is a pole of order 1 for $\mathcal{I}^{z}$.

Consider now the function $G$, given by

$$
G(z):=\Gamma(z+1-Q) \cdot \prod_{j=2, . ., h} \Gamma\left(z-\zeta_{j}\right) .
$$

If $S$ denotes the sphere

$$
S:=\left\{\left(u_{1}, u_{2}\right) \in \mathbb{R}^{2}: \rho\left(u_{1}, u_{2}\right)=1\right\}
$$


with surface measure $\sigma(S)$, set

$$
I^{z}\left(u_{1}, u_{2}\right):=\frac{G(0)\left(u_{1}^{2 n}+u_{2}^{2 m}\right)^{z-Q}}{\sigma(S) \Gamma(z) G(z)} .
$$

In the sequel we will denote by $\mathcal{S}\left(\mathbb{R}^{s}\right), s=2,3$, the Schwartz space on $\mathbb{R}^{s}$ endowed with a denumerable family of norms $\|\cdot\|_{(N)}$ given by

$$
\|\Phi\|_{(N)}=\sum_{|\alpha| \leq N} \sup _{u \in \mathbb{R}^{s}}(1+|u|)^{N}\left|\partial_{u}^{\alpha} \Phi(u)\right| .
$$

Here we use the conventional notation

$$
\partial_{u}^{\alpha}=\frac{\partial^{\alpha_{1}}}{\partial u_{1}^{\alpha_{1}}} \cdots \frac{\partial^{\alpha_{s}}}{\partial u_{s}^{\alpha_{s}}},
$$

with $\alpha=\left(\alpha_{1}, \ldots, \alpha_{s}\right)$ s-tuple of natural numbers and $|\alpha|=\alpha_{1}+\cdots+\alpha_{s}$.

Proposition 2.6. The distribution $I^{z}$ satisfies

$$
I^{0}=\delta_{0} \text {. }
$$

Proof. Take $z \in \mathbb{C}, \Re e z>0$, and $\varphi \in \mathcal{S}\left(\mathbb{R}^{2}\right)$. Set $C_{z}:=\frac{G(0)}{\sigma(S) \Gamma(z) G(z)}$. Then

$$
\begin{aligned}
<I^{z}, \varphi>= & C_{z} \int_{\mathbb{R}^{2}} \rho\left(u_{1}, u_{2}\right)^{z-Q} \varphi\left(u_{1}, u_{2}\right) d u_{1} d u_{2} \\
= & C_{z}\left(\int_{\left\{\rho\left(u_{1}, u_{2}\right) \leq 1\right\}} \rho\left(u_{1}, u_{2}\right)^{z-Q}\left(\varphi\left(u_{1}, u_{2}\right)-\varphi(0,0)\right) d u_{1} d u_{2}\right. \\
& +\int_{\left\{\rho\left(u_{1}, u_{2}\right) \leq 1\right\}} \rho\left(u_{1}, u_{2}\right)^{z-Q} \varphi(0,0) d u_{1} d u_{2} \\
& \left.+\int_{\left\{\rho\left(u_{1}, u_{2}\right) \geq 1\right\}} \rho\left(u_{1}, u_{2}\right)^{z-Q} \varphi\left(u_{1}, u_{2}\right) d u_{1} d u_{2}\right) \\
= & C_{z}\left(I_{1}+I_{2}+I_{3}\right) .
\end{aligned}
$$

By introducing polar coordinates (see [5]) we obtain

$$
\begin{aligned}
I_{2} & =\int_{S} \int_{0}^{1} \varphi(0,0) \rho\left(r \circ\left(v_{1}, v_{2}\right)\right)^{z-Q} r^{Q-1} d r d \sigma\left(v_{1} v_{2}\right) \\
& =\int_{S} \int_{0}^{1} \varphi(0,0)\left(r \cdot \rho\left(v_{1}, v_{2}\right)\right)^{z-Q} r^{Q-1} d r d \sigma\left(v_{1} v_{2}\right) \\
& =\varphi(0,0) \int_{S} \int_{0}^{1} r^{z-1} d r d \sigma\left(v_{1} v_{2}\right)=\varphi(0,0) \frac{\sigma(S)}{z},
\end{aligned}
$$

so that

$$
C_{z} I_{2}=\varphi(0,0) \frac{G(0)}{z \Gamma(z) G(z)}=\varphi(0,0) \frac{G(0)}{\Gamma(z+1) G(z)}
$$


and this expression is well-defined for every $z$, with $\Re e z>-\min \left\{-\zeta_{2}, 1\right\}$. Now, it it is easy to show that both $I_{1}$ and $I_{3}$ are absolutely convergent for $\Re e z>-\min \left\{\frac{1}{2 n},-\zeta_{2}\right\}$. Indeed,

$$
\begin{aligned}
\left|I_{1}\right| & \leq \int_{\left\{\rho\left(u_{1}, u_{2}\right) \leq 1\right\}} \rho\left(u_{1}, u_{2}\right)^{\Re e z-Q}\left|\varphi\left(u_{1}, u_{2}\right)-\varphi(0,0)\right| d u_{1} d u_{2} \\
& \leq C|| \nabla \varphi \|_{\infty} \int_{\left\{\rho\left(u_{1}, u_{2}\right) \leq 1\right\}} \rho\left(u_{1}, u_{2}\right)^{\Re e z-Q}\left|\left(u_{1}, u_{2}\right)\right| d u_{1} d u_{2} \\
& \leq C|| \nabla \varphi \|_{\infty} \int_{\left\{\rho\left(u_{1}, u_{2}\right) \leq 1\right\}} \rho\left(u_{1}, u_{2}\right)^{\Re e z-Q+\frac{1}{2 n}} d u_{1} d u_{2} \\
& =C\|\nabla \varphi\|_{\infty} \int_{S} \int_{0}^{1} \rho\left(r \circ\left(v_{1}, v_{2}\right)\right)^{\Re e z-Q+\frac{1}{2 n}} r^{Q-1} d r d \sigma\left(v_{1}, v_{2}\right) \\
& =C\|\nabla \varphi\|_{\infty} \int_{S} \int_{0}^{1} r^{\Re e z-Q+\frac{1}{2 n}} r^{Q-1} d r d \sigma\left(v_{1}, v_{2}\right) \\
& =C\|\nabla \varphi\|_{\infty} \frac{\sigma(S)}{\frac{1}{2 n}+\Re e z}
\end{aligned}
$$

which is well-defined for $\Re e z>-\frac{1}{2 n}$. Here, in particular, we used (2.3).

Moreover,

$$
\begin{aligned}
\left|I_{3}\right| & \leq \int_{\left\{\rho\left(u_{1}, u_{2}\right)>1\right\}} \rho\left(u_{1}, u_{2}\right)^{\Re e z-Q}\left|\varphi\left(u_{1}, u_{2}\right)\right| d u_{1} d u_{2} \\
& \leq C\|\varphi\|_{(N)} \int_{\left\{\rho\left(u_{1}, u_{2}\right)>1\right\}} \rho\left(u_{1}, u_{2}\right)^{\Re e z-Q-\frac{N}{2 n}} d u_{1} d u_{2},
\end{aligned}
$$

since

$$
\left|\varphi\left(u_{1}, u_{2}\right)\right| \leq \frac{\|\varphi\|_{(N)}}{\left(1+\left|\left(u_{1}, u_{2}\right)\right|\right)^{N}} \leq \frac{\|\varphi\|_{(N)}}{\left|\left(u_{1}, u_{2}\right)\right|^{N}} \leq C \frac{\|\varphi\|_{(N)}}{\left(\rho\left(u_{1}, u_{2}\right)\right)^{\frac{N}{2 n}}}
$$

when $\rho\left(u_{1}, u_{2}\right)>1$, as a consequence of (2.2). Now by passing to polar coordinates we obtain

$$
\begin{aligned}
\left|I_{3}\right| & \leq C\|\varphi\|_{(N)} \int_{S} \int_{1}^{+\infty} \rho\left(r \circ\left(v_{1}, v_{2}\right)\right)^{\Re e z-Q-\frac{N}{2 n}} r^{Q-1} d r d \sigma\left(v_{1} v_{2}\right) \\
& =C\|\varphi\|_{(N)} \frac{\sigma(S)}{\frac{N}{2 n}-\Re e z}<+\infty
\end{aligned}
$$

if $N$ is a positive integer greater than $2 n \cdot \Re e z$. Thus, as a consequence of the uniqueness of the analytic continuation, the expression

$$
<I^{z}, \varphi>=C_{z}\left(I_{1}+I_{2}+I_{3}\right)
$$

defines the action of $I^{z}$ on a Schwartz function $\varphi$ in $\mathbb{R}^{2}$, for $\Re e z>-\min \left\{\frac{1}{2 n}\right.$, $\left.-\zeta_{2}, 1\right\}$, and by using the bounds for $I_{1}$ and $I_{3}$ and (2.10) one gets the thesis. 
Proposition 2.7. $\quad I^{z}$ is a homogeneous tempered distribution of degree $-Q+z$.

We recall that this means that for all $\varphi \in \mathcal{S}\left(\mathbb{R}^{2}\right)$ the following equality is satisfied

$$
<I^{z}, \varphi_{\delta}>=\delta^{z-Q}<I^{z}, \varphi>
$$

where

$$
\varphi_{\delta}\left(u_{1}, u_{2}\right):=\delta^{-Q} \varphi\left(\delta^{-1} \circ u\right)=\delta^{-Q} \varphi\left(\delta^{-\frac{1}{2 n}} u_{1}, \delta^{-\frac{1}{2 m}} u_{2}\right)
$$

Thus the Fourier transform of the (tempered and homogeneous) distribution $I^{z}$ is a well-defined distribution, homogeneous of degree $-Q-(z-Q)=-z$. Moreover, the following holds.

Proposition 2.8. $\widehat{I}^{z}$ agrees with a function $C^{\infty}\left(\mathbb{R}^{2} \backslash\{(0,0)\}\right)$ away from $(0,0)$. Moreover,

$$
\left|\widehat{I^{z}}(\xi)\right| \leq C \rho(\xi)^{-\Re e z}, \quad \text { for all } \xi \in \mathbb{R}^{2} \backslash\{(0,0)\} .
$$

Proof. It suffices to prove the statement for $0<\Re e z<Q$; indeed, the other cases can be treated by analytic continuation.

We first construct a partition of unity adapted to dyadic spherical shells. The procedure is standard and we briefly recall it only for the sake of completeness.

Let $\psi$ be a $C_{c}^{\infty}\left(\mathbb{R}^{2}\right)$ function, such that

(i) $0 \leq \psi\left(u_{1}, u_{2}\right) \leq 1$ for every $\left(u_{1}, u_{2}\right) \in \mathbb{R}^{2}$;

(ii) $\psi\left(u_{1}, u_{2}\right) \equiv 0$ if $\left(u_{1}, u_{2}\right) \notin C_{0}:=\left\{\left(u_{1}, u_{2}\right) \in \mathbb{R}^{2}: \frac{1}{4} \leq \rho\left(u_{1}, u_{2}\right) \leq 8\right\}$;

(iii) $\psi\left(u_{1}, u_{2}\right) \equiv 1$ if $\left(u_{1}, u_{2}\right) \in C_{1}:=\left\{\left(u_{1}, u_{2}\right): \frac{1}{2} \leq \rho\left(u_{1}, u_{2}\right) \leq 4\right\}$.

Define now for $\left(u_{1}, u_{2}\right) \in \mathbb{R}^{2} \backslash\{(0,0)\}$

$$
\Psi\left(u_{1}, u_{2}\right):=\sum_{j \in \mathbb{Z}} \psi\left(2^{j} \circ\left(u_{1}, u_{2}\right)\right)
$$

Since there is at most a finite number of nonzero terms in the sum $(2.12), \Psi$ is well-defined and strictly positive on $\mathbb{R}^{2} \backslash\{(0,0)\}$. Thus we may introduce the functions

$$
\eta\left(u_{1}, u_{2}\right):=\frac{\psi\left(u_{1}, u_{2}\right)}{\Psi\left(u_{1}, u_{2}\right)}
$$


It is easy to check that

$$
\sum_{j \in \mathbb{Z}} \eta\left(2^{j} \circ\left(u_{1}, u_{2}\right)\right)=1 \quad \text { for every }\left(u_{1}, u_{2}\right) \in \mathbb{R}^{2} \backslash\{(0,0)\} .
$$

Now using (2.14) we may write

$$
\begin{aligned}
I^{z}\left(u_{1}, u_{2}\right) & =C_{z} \rho\left(u_{1}, u_{2}\right)^{z-Q} \\
& =C_{z} \sum_{j \in \mathbb{Z}} \eta\left(2^{j} \circ\left(u_{1}, u_{2}\right)\right) \rho\left(2^{-j} \circ 2^{j} \circ\left(u_{1}, u_{2}\right)\right)^{z-Q} \\
& =C_{z} \sum_{j \in \mathbb{Z}} \eta\left(2^{j} \circ\left(u_{1}, u_{2}\right)\right) 2^{-j(z-Q)} \rho\left(2^{j} \circ\left(u_{1}, u_{2}\right)\right)^{z-Q} \\
& =C_{z} \sum_{j \in \mathbb{Z}} 2^{-j(z-Q)} f_{0}\left(2^{j} \circ\left(u_{1}, u_{2}\right)\right),
\end{aligned}
$$

where we set

$$
f_{0}\left(u_{1}, u_{2}\right):=\eta\left(u_{1}, u_{2}\right) \rho\left(u_{1}, u_{2}\right)^{z-Q} .
$$

Since

$$
\left(f_{0}\left(2^{j} \circ(\cdot, \cdot)\right)\right)^{\curlyvee}\left(\xi_{1}, \xi_{2}\right)=2^{-j Q} \widehat{f}_{0}\left(2^{-j} \circ\left(\xi_{1}, \xi_{2}\right)\right),
$$

we obtain formally

$$
\sum_{j \in \mathbb{Z}} 2^{-j(z-Q)}\left(f_{0}\left(2^{j} \circ(\cdot, \cdot)\right)\right)^{\curlyvee}\left(\xi_{1}, \xi_{2}\right)=\sum_{j \in \mathbb{Z}} 2^{-j z} \widehat{f}_{0}\left(2^{-j} \circ\left(\xi_{1}, \xi_{2}\right)\right) .
$$

This series is absolutely convergent, since if $\left(\xi_{1}, \xi_{2}\right) \neq(0,0)$ one has

$$
\begin{aligned}
& \left|\sum_{j \in \mathbb{Z}} 2^{-j z} \widehat{f}_{0}\left(2^{-j} \circ\left(\xi_{1}, \xi_{2}\right)\right)\right| \leq \sum_{j \in \mathbb{Z}} 2^{-j \Re e z}\left|\widehat{f}_{0}\left(2^{-j} \circ\left(\xi_{1}, \xi_{2}\right)\right)\right| \\
& \quad \leq\left(\sum_{2^{-j} \rho\left(\xi_{1}, \xi_{2}\right) \leq 1}+\sum_{2^{-j} \rho\left(\xi_{1}, \xi_{2}\right)>1}\right) 2^{-j \Re e z}\left|\widehat{f}_{0}\left(2^{-j} \circ\left(\xi_{1}, \xi_{2}\right)\right)\right| \\
& \quad \leq\left\|\widehat{f}_{0}\right\|_{(0)} \sum_{2^{-j} \rho\left(\xi_{1}, \xi_{2}\right) \leq 1} 2^{-j \Re e z}+\sum_{\rho\left(2^{-j} \circ\left(\xi_{1}, \xi_{2}\right)\right)>1} 2^{-j \Re e z} \frac{\left\|\widehat{f}_{0}\right\|_{(N)}}{\left(1+\left|\left(2^{-j} \circ\left(\xi_{1}, \xi_{2}\right)\right)\right|\right)^{N}} \\
& \quad \leq C\left\|\widehat{f}_{0}\right\|_{(0)} \rho(\xi)^{-\Re e z}+C \sum_{2^{-j} \rho\left(\xi_{1}, \xi_{2}\right)>1} 2^{-j \Re e z} \frac{\left\|\widehat{f}_{0}\right\|_{(N)}}{\left(\rho\left(2^{-j} \circ\left(\xi_{1}, \xi_{2}\right)\right)\right)^{\frac{N}{2 n}}} \\
& \leq C\left\|\widehat{f}_{0}\right\|_{(0)} \rho(\xi)^{-\Re e z}+\frac{C}{\left(\rho\left(\xi_{1}, \xi_{2}\right)\right)^{\frac{N}{2 n}}} \sum_{2^{-j} \rho\left(\xi_{1}, \xi_{2}\right)>1} 2^{-j \Re e z+j \frac{N}{2 n}} \\
& \leq C\left\|\widehat{f}_{0}\right\|_{(0)} \rho(\xi)^{-\Re e z}+\frac{C}{\left(\rho\left(\xi_{1}, \xi_{2}\right)\right)^{\frac{N}{2 n}}} \rho\left(\xi_{1}, \xi_{2}\right)^{-\Re e z+\frac{N}{2 n}} \leq C \rho\left(\xi_{1}, \xi_{2}\right)^{-\Re e z},
\end{aligned}
$$


where we used in particular the fact that

$$
\left|\left(u_{1}, u_{2}\right)\right| \geq C \rho\left(u_{1}, u_{2}\right)^{\frac{1}{2 n}} \text { for }\left|\left(u_{1}, u_{2}\right)\right|>1 .
$$

We set therefore

$$
v\left(\xi_{1}, \xi_{2}\right):=\sum_{j \in \mathbb{Z}} 2^{-j z} \widehat{f}_{0}\left(2^{-j} \circ\left(\xi_{1}, \xi_{2}\right)\right) \in \mathrm{七}^{\infty}\left(\mathbb{R}^{2}\right) .
$$

By the Dominated Convergence Theorem we obtain, given $\varphi \in \mathcal{S}\left(\mathbb{R}^{2}\right)$,

$$
\int_{\mathbb{R}^{2}} v \varphi=\sum_{j \in \mathbb{Z}} 2^{-j z} \int_{\mathbb{R}^{2}} \widehat{f}_{0}\left(2^{-j} \circ(\cdot, \cdot)\right) \varphi,
$$

that is

$$
v(\cdot, \cdot)=\sum_{j \in \mathbb{Z}} 2^{-j z} \widehat{f}_{0}\left(2^{-j} \circ(\cdot, \cdot)\right)
$$

in the sense of distributions, whence

$$
\widehat{I^{z}}(\cdot, \cdot)=\sum_{j \in \mathbb{Z}} 2^{-j z} \widehat{f}_{0}\left(2^{-j} \circ(\cdot, \cdot)\right)
$$

in the sense of distributions and, moreover,

$$
\left|\widehat{I^{z}}\left(\xi_{1}, \xi_{2}\right)\right| \leq \rho\left(\xi_{1}, \xi_{2}\right)^{-\Re e z} \text { for all }\left(\xi_{1} \xi_{2}\right) \in \mathbb{R}^{2} \backslash\{(0,0)\}
$$

(observe that this inequality could also be retrieved from the homogeneity). Finally we prove that $\widehat{I^{z}}$ agrees with a function $C^{\infty}\left(\mathbb{R}^{2} \backslash\{(0,0)\}\right)$ away from $(0,0)$. First of all, we observe that $f_{0}$ is in the Schwartz space, hence $\widehat{f}_{0}\left(2^{-j} \circ(\cdot, \cdot)\right)$ belongs to $\mathcal{C}^{\infty}\left(\mathbb{R}^{2}\right)$. Moreover, the following estimates hold:

$$
\begin{gathered}
\left|\partial_{\xi_{1}}^{k}\left(2^{-j z} \widehat{f}_{0}\left(2^{-j} \circ\left(\xi_{1}, \xi_{2}\right)\right)\right)\right| \leq C_{k} 2^{-j\left(\Re e z+\frac{k}{2 n}\right)} \text { for all }\left(\xi_{1}, \xi_{2}\right) \in \mathbb{R}^{2} \\
\left|\partial_{\xi_{1}}^{k}\left(2^{-j z} \widehat{f}_{0}\left(2^{-j} \circ\left(\xi_{1}, \xi_{2}\right)\right)\right)\right| \leq C_{k, N} 2^{-j\left(\Re e z+\frac{k}{2 n}\right)} \frac{1}{\left(1+\left|\left(2^{-j} \circ\left(\xi_{1}, \xi_{2}\right)\right)\right|\right)^{N}} \\
\leq \frac{C_{k, N}}{\rho\left(\xi_{1}, \xi_{2}\right)^{\frac{N}{2 n}}} 2^{j\left(-\Re e z-\frac{k}{2 n}+\frac{N}{2 n}\right)} \quad \text { if } \rho\left(2^{-j} \circ\left(\xi_{1}, \xi_{2}\right)\right)>1,
\end{gathered}
$$

for all $k \in \mathbb{N}$. Since analogous bounds hold for $\partial_{\xi_{2}}^{k}\left(2^{-j z} \widehat{f}_{0}\left(2^{-j} \circ\left(\xi_{1}, \xi_{2}\right)\right)\right.$, with $2 n$ replaced by $2 m$, the series of the partial derivatives of $2^{-j z} \widehat{f}_{0}\left(2^{-j} \circ(\cdot, \cdot)\right)$ of any order $k$ converge on the compact subsets of $\mathbb{R}^{2} \backslash\{(0,0)\}$. It follows that $\widehat{I^{z}}(\cdot, \cdot)=\sum_{j \in \mathbb{Z}} 2^{-j z} \widehat{f}_{0}\left(2^{-j} \circ(\cdot, \cdot)\right)$ is $\mathcal{C}^{\infty}\left(\mathbb{R}^{2} \backslash\{(0,0)\}\right)$. 


\section{Some preliminary results}

In the following, if $f\left(x_{1}, x\right) \in \mathcal{S}\left(\mathbb{R}^{3}\right)$ we denote by $\mathcal{F}^{-1} f$ the inverse Fourier transform of $f$ and by $\mathcal{F}_{2} f$ and $\mathcal{F}_{2}^{-1} f$ respectively the partial Fourier transform and the inverse of the partial Fourier transform of $f$ with respect to the variable $x$.

Moreover we denote the dual variables as $\left(\xi_{1}, \xi\right)$ with $\xi=\left(\xi_{2}, \xi_{3}\right)$.

\section{Characterization of product kernels}

As recalled in the Introduction, the precise definition of product kernels involves certain differential inequalities and certain cancellation conditions which are analogous to those satisfied by the kernel $H\left(x_{1}, x\right)$ defined by (1.3). Our study will be based on the following equivalent definition (see [12]).

Definition 3.1. A product kernel $K$ on $\mathbb{R}^{3}$ is a sum

$$
K\left(x_{1}, x\right)=\sum_{J \in \mathbb{Z}^{2}} 2^{-j_{1}-j Q} \psi_{J}\left(2^{-j_{1}} x_{1}, 2^{-j} \circ x\right), \quad J=\left(j_{1}, j\right)
$$

convergent in the sense of distributions, of smooth functions $\psi_{J}$ supported on the set where $1 / 2 \leq\left|x_{1}\right| \leq 4$ and $1 / 2 \leq \rho(x) \leq 4$, satisfying the cancellation conditions

$$
\begin{aligned}
& \int \psi_{J}\left(x_{1}, x\right) d x_{1}=0 \\
& \int \psi_{J}\left(x_{1}, x\right) d x=0
\end{aligned}
$$

identically for every $J$, and with uniformly bounded $C^{k}$ norms for every $k \in \mathbb{N}$.

We shall need a characterization of product kernels as dyadic sums of Schwartz functions on $\mathbb{R}^{3}$ which are compactly supported only in the first variable and that satisfy some moment conditions.

Lemma 3.2. A product kernel $K$ on $R^{3}$ can be written as a sum

$$
K\left(x_{1}, x\right)=\sum_{J \in \mathbb{Z}^{2}} 2^{-j_{1}-j Q} \varphi_{J}\left(2^{-j_{1}} x_{1}, 2^{-j} \circ x\right), \quad J=\left(j_{1}, j\right),
$$

convergent in the sense of distributions, of Schwartz functions $\varphi_{J}$ such that

(i) the $\varphi_{J}$ have compact $x_{1}$-support where $1 / 2 \leq\left|x_{1}\right| \leq 4$;

(ii) the $\varphi_{J}$ form a bounded set of $\mathcal{S}\left(\mathbb{R}^{3}\right)$, that is the Schwartz norms $\left\|\varphi_{J}\right\|_{(N)}$ are uniformly bounded in $J$ for each $N \in \mathbb{N}$; 
(iii) the $\varphi_{J}$ satisfy the cancellation conditions

$$
\int x_{1}^{\ell} \varphi_{J}\left(x_{1}, x\right) d x_{1}=0
$$

for every positive integer $\ell \leq M_{1}$, for some fixed $M_{1} \in \mathbb{N}$, and

$$
\int x^{\beta} \varphi_{J}\left(x_{1}, x\right) d x=0
$$

for every multi-index $\beta=\left(\beta_{1}, \beta_{2}\right) \in \mathbb{N}^{2}$, identically for every $J \in \mathbb{Z}^{2}$. Here, as usual, $x^{\beta}=x_{2}^{\beta_{1}} x_{3}^{\beta_{2}}$.

Proof. Let $K$ be a product kernel on $\mathbb{R}^{3}$. By Definition 3.1 we can write $K$ as a sum

$$
K\left(x_{1}, x\right)=\sum_{\left(j_{1}, i\right) \in \mathbb{Z}^{2}} 2^{-j_{1}-i Q} \psi_{\left(j_{1}, i\right)}\left(2^{-j_{1}} x_{1}, 2^{-i} \circ x\right),
$$

convergent in the sense of distributions, of smooth functions $\psi_{\left(j_{1}, i\right)}$ supported on the set where $1 / 2 \leq\left|x_{1}\right| \leq 4$ and $1 / 2 \leq \rho(x) \leq 4$, satisfying the cancellation conditions (3.2) and (3.3) identically for every $\left(j_{1}, i\right) \in \mathbb{Z}^{2}$, and with uniformly bounded $C^{k}$ norms for every $k \in \mathbb{N}$.

Let

$$
\widehat{K}\left(\xi_{1}, \xi\right)=\sum_{\left(j_{1}, i\right) \in \mathbb{Z}^{2}} \widehat{\psi}_{\left(j_{1}, i\right)}\left(2^{j_{1}} \xi_{1}, 2^{i} \circ \xi\right)
$$

be the corresponding product multiplier.

Consider a smooth function $\zeta$ on the real line, supported on the interval $[1,4]$ and such that $\sum_{k \in \mathbb{Z}} \zeta\left(2^{k} t\right)=1$ for every $t>0$. For $J=\left(j_{1}, j\right) \in \mathbb{Z}^{2}$, define

$$
\mu_{J}\left(\xi_{1}, \xi\right):=\sum_{i \in \mathbb{Z}} \widehat{\psi}_{\left(j_{1}, i\right)}\left(\xi_{1}, 2^{i-j} \circ \xi\right) \zeta(\rho(\xi)) .
$$

It can be easily proved that the $\mu_{J}$ form a bounded set of $\mathcal{S}\left(\mathbb{R}^{3}\right)$. In addition, a direct computation shows that

$$
\widehat{K}\left(\xi_{1}, \xi\right)=\sum_{J \in \mathbb{Z}^{2}} \mu_{J}\left(2^{j_{1}} \xi_{1}, 2^{j} \circ \xi\right)
$$

in the sense of distributions. Setting

$$
\varphi_{J}\left(x_{1}, x\right):=\left(\mathcal{F}^{-1} \mu_{J}\right)\left(x_{1}, x\right)=\mathcal{F}_{2}^{-1}\left(\sum_{i \in \mathbb{Z}}\left(\mathcal{F}_{2} \psi_{\left(j_{1}, j\right)}\right)\left(x_{1}, 2^{i-j} \circ \cdot\right) \zeta(\rho(\cdot))\right)(x),
$$

it is possible to write the product kernel $K$ as the sum

$$
K\left(x_{1}, x\right)=\sum_{J \in \mathbb{Z}^{2}} 2^{-j_{1}-j Q} \varphi_{J}\left(2^{-j_{1}} x_{1}, 2^{-j} \circ x\right),
$$


convergent in the sense of distributions, of functions $\varphi_{J}$ that form a bounded set of $\mathcal{S}\left(\mathbb{R}^{3}\right)$ and have compact $x_{1}$-support where $1 / 2 \leq\left|x_{1}\right| \leq 4$. Finally, the fact that $\mu_{J}(0, \xi)=0$ and $\left(\partial_{\xi}^{\beta} \mu_{J}\right)\left(\xi_{1}, 0\right)=0$ for every multi-index $\beta=$ $\left(\beta_{1}, \beta_{2}\right) \in \mathbb{N}^{2}$, identically for every $J \in \mathbb{Z}^{2}$, yields (3.4) for $m=0$ and (3.5).

In fact, we can choose $\varphi_{J}$ so that a finite number of moments in the variable $x_{1}$ vanish. This follows from a slight modification of the arguments in Lemma 2.2.3 in [12]. More explicitly, denote by $\varphi$ each function $\varphi_{J}$ in the decomposition (3.6). Then each function $\varphi$ may be written as a series

$$
\varphi\left(x_{1}, x\right)=\sum_{k \in \mathbb{Z}} 2^{-k} A_{k}\left(2^{-k} x_{1}, x\right),
$$

convergent in the sense of distributions, of functions $A_{k}$ which form a bounded set of $\mathcal{S}\left(\mathbb{R}^{3}\right)$ with norms that decay exponentially in $k$ as $k \rightarrow \pm \infty$, have compact $x_{1}$-support on the set $\left\{x_{1} \in \mathbb{R}: 1 / 2 \leq\left|x_{1}\right| \leq 4\right\}$, and satisfy (3.4) with $\ell=1$.

To prove this fact, consider a function $\eta \in \mathcal{C}_{0}^{\infty}(\mathbb{R})$, supported on the set $[-4,-1] \cup[1,4]$, such that $\sum_{k \in \mathbb{Z}} \eta\left(2^{k} t\right)=1$ for every $t \neq 0$ and $\int t \eta(t) d t \neq 0$. Set

$$
\begin{aligned}
\chi_{k}\left(x_{1}\right) & :=\eta\left(2^{-k} x_{1}\right), \\
\tilde{\chi}_{k}\left(x_{1}\right) & :=\frac{\chi_{k}\left(x_{1}\right)}{\int x_{1} \chi_{k}\left(x_{1}\right) d x_{1}}, \\
a_{k}(x) & =\int x_{1} \chi_{k}\left(x_{1}\right) \varphi\left(x_{1}, x\right) d x_{1}, \\
S_{k}(x) & =\sum_{j \geq k} a_{j}(x) .
\end{aligned}
$$

Then write $\varphi$ as

$$
\begin{aligned}
\varphi\left(x_{1}, x\right) & =\sum_{k \in \mathbb{Z}}\left(\varphi\left(x_{1}, x\right) \chi_{k}\left(x_{1}\right)-a_{k}(x) \tilde{\chi}_{k}\left(x_{1}\right)\right)+\sum_{k \in \mathbb{Z}}\left(S_{k}(x)-S_{k+1}(x)\right) \tilde{\chi}_{k}\left(x_{1}\right) \\
& =\sum_{k \in \mathbb{Z}}\left(\varphi\left(x_{1}, x\right) \chi_{k}\left(x_{1}\right)-a_{k}(x) \tilde{\chi}_{k}\left(x_{1}\right)\right)+\sum_{k \in \mathbb{Z}} S_{k}(x)\left(\tilde{\chi}_{k}\left(x_{1}\right)-\tilde{\chi}_{k-1}\left(x_{1}\right)\right) \\
& =\sum_{k \in \mathbb{Z}} A_{k}\left(x_{1}, x\right),
\end{aligned}
$$

where the series converges in the sense of distributions, the functions $A_{k}$ satisfy the moment conditions $\int x_{1} A_{k}\left(x_{1}, x\right) d x_{1}=0$ for all $k \in \mathbb{Z}$, and the Schwartz norms decay exponentially in $k$ as $k \rightarrow \pm \infty$. Now by rescaling $x_{1}$ we obtain (3.7). Iterating this argument yields (3.4) for all $\ell \leq M_{1}$, for some fixed $M_{1} \in \mathbb{N}$.

A result analogous to Lemma 3.2 can be stated by interchanging the role of $x_{1}$ and $x$. 


\section{Estimates on oscillatory integrals}

In the following, we prove some estimates on certain oscillatory integrals related to our problem. Let ||$|\cdot|||$ denote any homogeneous norm with respect to the family of non-isotropic dilations

$$
\delta \bullet\left(\xi_{1}, \xi\right)=\left(\delta \xi_{1}, \delta^{m} \xi_{2}, \delta^{n} \xi_{3}\right), \quad \delta>0,
$$

e.g. we may choose

$$
\|\left|\left(\xi_{1}, \xi\right)\right|||=\max \left\{\left|\xi_{1}\right|,\left|\xi_{2}\right|^{\frac{1}{m}},\left|\xi_{3}\right|^{\frac{1}{n}}\right\} .
$$

We observe in passing that

$$
\delta \bullet\left(\xi_{1}, \xi\right)=\left(\delta \xi_{1}, \delta^{2 m n} \circ \xi\right) .
$$

Consider the integral

$$
I\left(\xi_{1}, \xi, \eta\right):=\int f\left(x_{1}, \eta\right) e^{-i\left(\xi_{1}, \xi\right) \cdot\left(x_{1}, \gamma\left(x_{1}\right)\right)} d x_{1},
$$

where $\gamma\left(x_{1}\right)=\left(x_{1}^{m}, x_{1}^{n}\right)$ and $f$ is such that

(h1) $f$ belongs to $\mathcal{S}\left(\mathbb{R}^{3}\right)$ and is $x_{1}$-compactly supported on the interval $1 / 2 \leq\left|x_{1}\right| \leq 4$

(h2) $f\left(x_{1}, 0\right)=0$ for all $x_{1}$ such that $1 / 2 \leq\left|x_{1}\right| \leq 4$.

The constants $C_{N}$ occurring in the following inequalities depend on the Schwartz norms of $f$.

Lemma 3.3. Under the hypotheses (h1) and (h2) the following estimate holds for the integral $I\left(\xi_{1}, \xi, \eta\right)$ defined by (3.11)

$$
\left|I\left(\xi_{1}, \xi, \eta\right)\right| \leq C_{N} \frac{\rho(\eta)^{\frac{1}{2 n}}}{(1+\rho(\eta))^{N}}
$$

for every integer $N \geq 0$.

Proof. Since $f$ is a Schwartz function, by using (h2), (2.2) and (2.3) we deduce that for every $\left(x_{1}, \eta\right) \in\left\{x_{1} \in \mathbb{R}: 1 / 2 \leq\left|x_{1}\right| \leq 4\right\} \times \mathbb{R}^{2}$

$$
\left|\partial_{x_{1}}^{k} f\left(x_{1}, \eta\right)\right| \leq C_{N} \frac{\rho(\eta)^{\frac{1}{2 n}}}{(1+\rho(\eta))^{N}}
$$

for all $N \in \mathbb{N}$ and $k \in \mathbb{N}$. For $k=0$ this inequality yields then (3.12). 
Lemma 3.4. Let I be the oscillatory integral defined by (3.11). Assume that (h1) and (h2) are satisfied. Then

$$
\left|I\left(\xi_{1}, \xi, \eta\right)\right| \leq C_{N} \frac{\rho(\eta)^{\frac{1}{2 n}}}{\left.(1+\rho(\eta))^{N}||\left|\left(\xi_{1}, \xi\right)\right|\right|^{\frac{1}{n}}} \quad \text { if } \quad||\left|\left(\xi_{1}, \xi\right)\right|||>1
$$

for every integer $N \geq 0$.

Under the additional assumption

$$
\int f\left(x_{1}, x\right) d x_{1}=0, x \in \mathbb{R}^{2},
$$

the following estimate holds

$$
\left|I\left(\xi_{1}, \xi, \eta\right)\right| \leq C_{N} \frac{\rho(\eta)^{\frac{1}{2 n}}}{(1+\rho(\eta))^{N}}\left|\left\|\left(\xi_{1}, \xi\right)|\|| \quad \text { if } \quad\right\|\right|\left(\xi_{1}, \xi\right) \mid \| \leq 1
$$

for every integer $N \geq 0$.

Proof. Let ||$\left(\xi_{1}, \xi\right)|| \mid$ be the homogeneous norm defined in (3.9). We divide the phase space $\xi_{1}, \xi$ into two regions, depending on whether $\left\|\left|\left(\xi_{1}, \xi\right)\right|\right\| \leq 1$ or $\left\|||\left(\xi_{1}, \xi\right)|\||>1\right.$.

Assume that $\left\|||\left(\xi_{1}, \xi\right)\right\|||>1$ and write $\left(\xi_{1}, \xi\right)=\lambda \omega$, where $\lambda=\|\|\left(\xi_{1}, \xi\right)|\||$ and $\omega=\left(\xi_{1}, \xi\right) /\left\|\mid\left(\xi_{1}, \xi\right)\right\| \|$.

With these notation, the oscillatory integral $I\left(\xi_{1}, \xi, \eta\right)$ becomes

$$
I(\lambda \omega, \eta)=\int_{\frac{1}{2} \leq\left|x_{1}\right| \leq 4} f\left(x_{1}, \eta\right) e^{-i \lambda \omega \cdot\left(x_{1}, \gamma\left(x_{1}\right)\right)} d x_{1} .
$$

Set

$$
\Phi\left(x_{1}\right)=\omega \cdot\left(x_{1}, \gamma\left(x_{1}\right)\right)=\frac{1}{\|\|\left(\xi_{1}, \xi\right) \mid \|}\left(\xi_{1} x_{1}+\xi_{2} x_{1}^{m}+\xi_{3} x_{1}^{n}\right),
$$

for $1 / 2 \leq\left|x_{1}\right| \leq 4$

We observe that, since the curve $x_{1} \mapsto\left(x_{1}, x_{1}^{m}, x_{1}^{n}\right)$ for $1 / 2 \leq\left|x_{1}\right| \leq 4$ is of finite type $\mathrm{n}$, for every $x_{1} \in \mathbb{R}$ with $1 / 2 \leq\left|x_{1}\right| \leq 4$ there exists a positive integer $\bar{n} \leq n$ such that $\Phi^{(\bar{n})}\left(x_{1}\right) \neq 0[15]$.

A standard application of the Van der Corput's lemma to the oscillatory integral (3.17), the compactness of the $x_{1}$-support , and

$$
\left|\Phi^{(\bar{n})}\left(x_{1}\right)\right| \geq C
$$

for some $C>0$, for all $x_{1}$ such that $\frac{1}{4} \leq\left|x_{1}\right| \leq 4$, yield

$$
|I(\lambda \omega, \eta)| \leq C \lambda^{-\frac{1}{n}}\left\|\partial_{x_{1}} f\left(x_{1}, \eta\right)\right\|_{L^{1}\left(\mathbb{R}_{x_{1}}\right)} .
$$


Since the function $f\left(x_{1}, \eta\right)$ is smooth and compactly supported in $x_{1}$ where $\frac{1}{2} \leq\left|x_{1}\right| \leq 4$, by using the estimate (3.13) in the case $k=1$ we see that

$$
\left\|\partial_{x_{1}} f\left(x_{1}, \eta\right)\right\|_{L^{1}\left(\mathbb{R}_{x_{1}}\right)} \leq C_{N} \frac{\rho(\eta)^{\frac{1}{2 n}}}{(1+\rho(\eta))^{N}}
$$

for every $N \in \mathbb{N}$. As a consequence,

$$
|I(\lambda \omega, \eta)| \leq C_{N} \lambda^{-\frac{1}{n}} \frac{\rho(\eta)^{\frac{1}{2 n}}}{(1+\rho(\eta))^{N}}
$$

for every $N \in \mathbb{N}$. Since $\lambda=\left\|\mid\left(\xi_{1}, \xi\right)\right\| \|$, we get (3.14).

Assume now that $\left|\left\|\left(\xi_{1}, \xi\right) \mid\right\| \leq 1\right.$. The cancellation condition (3.15), the Mean value theorem and the estimate (3.13) can be used to prove that

$$
\begin{aligned}
\left|I\left(\xi_{1}, \xi, \eta\right)\right| & \leq \int_{\frac{1}{2} \leq\left|x_{1}\right| \leq 4}\left|f\left(x_{1}, \eta\right)\right|\left|e^{-i\left(\xi_{1}, \xi\right) \cdot\left(x_{1}, \gamma\left(x_{1}\right)\right)}-1\right| d x_{1} \\
& \leq C_{N} \frac{\rho(\eta)^{\frac{1}{2 n}}}{(1+\rho(\eta))^{N}}\left|\left(\xi_{1}, \xi\right)\right|, \quad \text { for every } N \in \mathbb{N} .
\end{aligned}
$$

Since by hypothesis ||$\left|\left(\xi_{1}, \xi\right)\right||| \leq 1$, we have that $\left|\left(\xi_{1}, \xi\right)\right| \leq 3||\left|\left(\xi_{1}, \xi\right)\right|||$. This inequality, together with the previous estimate, yields (3.16).

The estimate (3.14) in Lemma 3.4 can be improved in the region of the space $\left(\xi_{1}, \xi\right)$ where the first derivative of the phase (3.18) never vanishes for $1 / 2 \leq\left|x_{1}\right| \leq 4$, as the following lemma shows.

Lemma 3.5. Let I be the oscillatory integral defined by (3.11). Under the hypotheses (h1) and (h2) there exists a constant $\tilde{C}>1$ such that for every integer $N \geq 0$

$$
\left|I\left(\xi_{1}, \xi, \eta\right)\right| \leq C_{N} \frac{\rho(\eta)^{\frac{1}{2 n}}}{\left.(1+\rho(\eta))^{N}||\left|\left(\xi_{1}, \xi\right)\right|\right|^{N}} \quad \text { when } \quad\left\|\left|\left(\xi_{1}, \xi\right)\right|\right\| \mid>1
$$

and

$$
\left|\xi_{1}\right|>\tilde{C}\left(\left|\xi_{2}\right|+\left|\xi_{3}\right|\right), \text { or }\left|\xi_{2}\right|>\tilde{C}\left(\left|\xi_{1}\right|+\left|\xi_{3}\right|\right) \text {, or }\left|\xi_{3}\right|>\tilde{C}\left(\left|\xi_{1}\right|+\left|\xi_{2}\right|\right) \text {. }
$$

Proof. We use for the integral $I$ the notation introduced in formula (3.17).

In order to improve the estimate (3.14), we have to determine the subsets of the phase space $\left(\xi_{1}, \xi\right)$ where

$$
\Phi^{\prime}\left(x_{1}\right)=\xi_{1}+m \xi_{2} x_{1}^{m-1}+n \xi_{3} x_{1}^{n-1}=0
$$


for some $x_{1} \in \mathbb{R}$ such that $\frac{1}{2} \leq\left|x_{1}\right| \leq 4$. Some elementary estimates show that we can find a constant $\tilde{C}>1$ sufficiently large, such that for any fixed point $\left(\xi_{1}, \xi\right) \in \mathbb{R}^{3}$, satisfying $\left\|\mid\left(\xi_{1}, \xi\right)\right\| \|>1$ and

$$
\left|\xi_{1}\right|>\tilde{C}\left(\left|\xi_{2}\right|+\left|\xi_{3}\right|\right), \text { or }\left|\xi_{2}\right|>\tilde{C}\left(\left|\xi_{1}\right|+\left|\xi_{3}\right|\right), \text { or }\left|\xi_{3}\right|>\tilde{C}\left(\left|\xi_{1}\right|+\left|\xi_{2}\right|\right),
$$

there exists a constant $C_{\omega}>0$ such that

$$
\left|\Phi^{\prime}\left(x_{1}\right)\right| \geq C_{\omega}
$$

for every $x_{1} \in \mathbb{R}$ with $1 / 2 \leq\left|x_{1}\right| \leq 4$.

Let $D$ denote the differential operator

$$
D f\left(x_{1}, \eta\right)=\left(-i \lambda \Phi^{\prime}\left(x_{1}\right)\right)^{-1} \frac{\partial f}{\partial x_{1}}\left(x_{1}, \eta\right)
$$

and let ${ }^{t} D$ denote its transpose

$$
{ }^{t} D f\left(x_{1}, \eta\right)=\frac{\partial}{\partial x_{1}}\left(\frac{f}{i \lambda \Phi^{\prime}\left(x_{1}\right)}\right) .
$$

Since $D^{N}\left(e^{-i \lambda \Phi\left(x_{1}\right)}\right)=e^{-i \lambda \Phi\left(x_{1}\right)}$ for every $N \in \mathbb{N}$, integration by parts shows that

$$
\begin{aligned}
I(\lambda \omega, \eta) & =\int_{\frac{1}{2} \leq\left|x_{1}\right| \leq 4} f\left(x_{1}, \eta\right) D^{N}\left(e^{-i \lambda \Phi\left(x_{1}\right)}\right) d x_{1} \\
& =\int_{\frac{1}{2} \leq\left|x_{1}\right| \leq 4}\left({ }^{t} D\right)^{N} f\left(x_{1}, \eta\right) e^{-i \lambda \Phi\left(x_{1}\right)} d x_{1} .
\end{aligned}
$$

Since $f\left(x_{1}, \eta\right)$ is a smooth function with compact support in the $x_{1}$ variable in the region where $1 / 2 \leq\left|x_{1}\right| \leq 4, f$ satisfies the estimate (3.13), and $\Phi\left(x_{1}\right)$ is a smooth function satisfying the inequality (3.23), we can verify that

$$
\left|\left({ }^{t} D\right)^{N} f\left(x_{1}, \eta\right)\right| \leq C_{N, \omega} \frac{\rho(\eta)^{\frac{1}{2 n}}}{(1+\rho(\eta))^{N}} \lambda^{-N}
$$

for every $N \in \mathbb{N}$.

Therefore we conclude that

$$
|I(\lambda \omega, \eta)| \leq C_{N, \omega} \lambda^{-N} \frac{\rho(\eta)^{\frac{1}{2 n}}}{(1+\rho(\eta))^{N}}
$$

for every $N \in \mathbb{N}$. By a compactness argument we can show that the previous estimate is independent of $\omega$, so that

$$
|I(\lambda \omega, \eta)| \leq C_{N} \lambda^{-N} \frac{\rho(\eta)^{\frac{1}{2 n}}}{(1+\rho(\eta))^{N}}
$$

for every $N \in \mathbb{N}$. Since $\lambda=\left\|\mid\left(\xi_{1}, \xi\right)\right\| \|$, we obtain the inequality (3.21). 
Remark 3.6. In the sequel, we shall systematically apply the estimates (3.12), (3.14), (3.16), and (3.21) to the oscillatory integral (3.11) with the integrand $f\left(x_{1}, \eta\right)$ of the form $x_{1}^{\alpha}\left(\mathcal{F}_{2}\left(x^{\beta} \varphi_{J}\right)\right)\left(x_{1}, \eta\right)$, where the functions $\varphi_{J}$ are given by Lemma 3.2. In particular, the Schwartz norms $\|\cdot\|_{(N)}$ of the functions $\varphi_{J}$ are uniformly bounded in $J \in \mathbb{Z}^{2}$ for every $N \in \mathbb{N}$.

We observe that the functions $x_{1}^{\alpha}\left(\mathcal{F}_{2}\left(x^{\beta} \varphi_{J}\right)\right)\left(x_{1}, \eta\right)$ fulfill the hypotheses (h1) and (h2), as a consequence of the cancellation condition (3.5).

Moreover we have

$$
\int x_{1}^{\ell}\left(\mathcal{F}_{2}\left(x^{\beta} \varphi_{J}\right)\right)\left(x_{1}, \eta\right) d x_{1}=0
$$

as a consequence of the cancellation property (3.4) for all $\ell \leq M_{1}$ for some fixed $M_{1} \in \mathbb{N}$, so that (3.15) is satisfied.

\section{4. $L^{2}$-boundedness}

Let $K$ be the kernel defined by (1.4) and $T$ the operator given by $T: f \mapsto$ $f * K$. In this section we prove that $T$ is bounded on $L^{2}\left(\mathbb{R}^{3}\right)$.

Let $J=\left(j_{1}, j\right)$. We proved in Lemma 3.2 that the product kernel $K_{0}$ can be written as a sum

$$
K_{0}\left(x_{1}, x\right)=\sum_{J \in \mathbb{Z}^{2}} 2^{-j_{1}-j Q} \varphi_{J}\left(2^{-j_{1}} x_{1}, 2^{-j} \circ x\right)
$$

convergent in the sense of distributions, of Schwartz functions $\left\{\varphi_{J}\right\}_{J \in \mathbb{Z}^{2}}$ on $\mathbb{R}^{3}$, satisfying the properties (i), (ii) and (iii) of Lemma 3.2.

Proposition 4.1. The series

$$
K\left(x_{1}, x\right)=\sum_{J \in \mathbb{Z}^{2}} 2^{-j_{1}-j Q} \varphi_{J}\left(2^{-j_{1}} x_{1}, 2^{-j} \circ\left(x-\gamma\left(x_{1}\right)\right)\right.
$$

converges in the sense of distributions and the corresponding convolution operator $T$ is bounded on $L^{2}\left(\mathbb{R}^{3}\right)$.

Proof. Let $m_{J}\left(\xi_{1}, \xi\right)$ be the Fourier transform of the $J$-th summand of the series (4.1). With a change of variables, we may write

$$
\begin{aligned}
m_{J}\left(\xi_{1}, \xi\right) & =\int_{1 / 2 \leq\left|x_{1}\right| \leq 4} \int \varphi_{J}\left(x_{1}, x\right) e^{-i\left(\xi_{1}, \xi\right) \cdot\left(2^{j_{1}} x_{1}, 2^{j} \circ x+\gamma\left(2^{j_{1}} x_{1}\right)\right)} d x_{1} d x \\
& =\int_{1 / 2 \leq\left|x_{1}\right| \leq 4}\left(\mathcal{F}_{2} \varphi_{J}\right)\left(x_{1}, 2^{j} \circ \xi\right) e^{-i\left(2^{j_{1}} \xi_{1}, 2^{2 m n} j_{1} \circ \xi\right) \cdot\left(x_{1}, \gamma\left(x_{1}\right)\right)} d x_{1} .
\end{aligned}
$$


Let $\|\left|\left(\xi_{1}, \xi\right)\right|||$ be any norm homogeneous with respect to the family of nonisotropic dilations (3.8), e.g. we may choose the norm defined by (3.9). We decompose the series

$$
\sum_{J \in \mathbb{Z}^{2}} m_{J}\left(\xi_{1}, \xi\right)
$$

as

$$
\sum_{2^{j_{1}}} m_{J}\left(\xi_{1}, \xi\right)+\sum_{\substack{\left(\xi_{1}, \xi\right)\|\| \mid \leq 1 \\ j \in \mathbb{Z}}} m_{J}\left(\xi_{1}, \xi\right) .
$$

It suffices to prove that each of the two series in (4.4) converges boundedly to a bounded function. To do this, we apply Lemma 3.4 to the oscillatory integral

$$
I_{J}\left(\xi_{1}, \xi, \eta\right):=\int_{\frac{1}{2} \leq\left|x_{1}\right| \leq 4}\left(\mathcal{F}_{2}\left(\varphi_{J}\right)\right)\left(x_{1}, \eta\right) e^{-i\left(\xi_{1}, \xi\right) \cdot\left(x_{1}, \gamma\left(x_{1}\right)\right)} d x_{1} .
$$

The functions $\left(\mathcal{F}_{2}\left(\varphi_{J}\right)\right)\left(x_{1}, \eta\right)$ have Schwartz norms $\left\|\varphi_{J}\right\|_{(N)}$ uniformly bounded in $J \in \mathbb{Z}^{2}$ for every $N \in \mathbb{N}$. As observed in Remark 3.6, they satisfy (h1), (h2) and (3.15), so that both estimates (3.14) and (3.16) hold, with costants $C_{N}$ independent of $N$.

More precisely, consider the first series in (4.4). Since

$$
m_{J}\left(\xi_{1}, \xi\right)=I_{J}\left(2^{j_{1}} \xi_{1}, 2^{2 m n j_{1}} \circ \xi, 2^{j} \circ \xi\right),
$$

by applying (3.16) we obtain

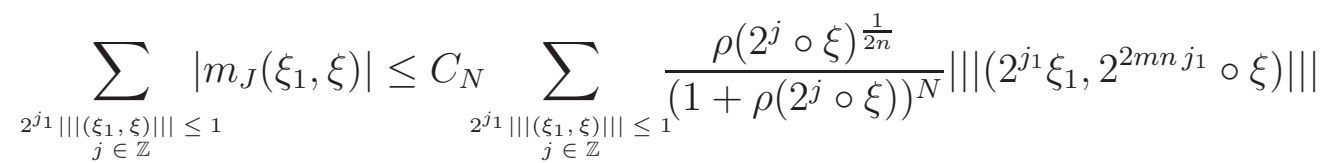

$$
\begin{aligned}
& =\sum_{\substack{2^{j_{1}}\left\|\left(\xi_{1}, \xi\right)\right\| \| \leq 1 \\
j \in \mathbb{Z}}} \frac{\left(2^{j} \rho(\xi)\right)^{\frac{1}{2 n}}}{\left(1+2^{j} \rho(\xi)\right)^{N}} 2^{j_{1}}\left|\left\|\left(\xi_{1}, \xi\right)|\||\right.\right. \\
& \leq C_{N} \sum_{j \in \mathbb{Z}} \frac{\left(2^{j} \rho(\xi)\right)^{\frac{1}{2 n}}}{\left(1+2^{j} \rho(\xi)\right)^{N}}
\end{aligned}
$$

for every $N \in \mathbb{N}$. Since the series

$$
\sum_{j \in \mathbb{Z}} \frac{\left(2^{j} b\right)^{\frac{1}{2 n}}}{\left(1+2^{j} b\right)^{N}}
$$

is uniformly bounded in $b$, it follows that the series in the previous formula converges boundedly to a bounded function. 
By using the identity (4.6) and the inequality (3.14) we prove that also the second sum on the right-hand side of (4.4) converges boundedly to a bounded function.

This proves that the series in (4.3) converges boundedly (and hence in the sense of distributions) to a bounded function $m\left(\xi_{1}, \xi\right)$. As a consequence the series (4.1) converges in the sense of distributions to the distribution $K=\mathcal{F}^{-1}(m)$.

Finally, by Plancherel's theorem, the boundedness of $m$ implies that the corresponding operator $T$ is bounded on $L^{2}\left(\mathbb{R}^{3}\right)$.

\section{5. $L^{p}$-boundedness}

In this section we prove the $L^{p}$-boundedness of the operator $T$. For this we split the sum (4.1) into two parts

$$
\begin{aligned}
K\left(x_{1}, x\right)= & \sum_{2 m n j_{1} \leq j} 2^{-j_{1}-j Q} \varphi_{J}\left(2^{-j_{1}} x_{1}, 2^{-j} \circ\left(x-\gamma\left(x_{1}\right)\right)\right. \\
& \quad+\sum_{2 m n j_{1}>j} 2^{-j_{1}-j Q} \varphi_{J}\left(2^{-j_{1}} x_{1}, 2^{-j} \circ\left(x-\gamma\left(x_{1}\right)\right)\right. \\
= & : K_{1}\left(x_{1}, x\right)+K_{2}\left(x_{1}, x\right) .
\end{aligned}
$$

Correspondingly we break the operator $T$ into the sum

$$
T f=f * K_{1}+f * K_{2}=: T_{1} f+T_{2} f
$$

and we prove that $T_{1}$ and $T_{2}$ are bounded on $L^{p}\left(\mathbb{R}^{3}\right)$ for $1<p<\infty$.

Proposition 5.1. The operator $T_{1}$ is bounded on $L^{p}\left(\mathbb{R}^{3}\right)$ for $1<p<\infty$.

Proof. Let $m_{J}\left(\xi_{1}, \xi\right)$ be the multiplier given in (4.2). We show that the series

$$
\widehat{K_{1}}\left(\xi_{1}, \xi\right)=\sum_{2 m n j_{1} \leq j} m_{J}\left(\xi_{1}, \xi\right)
$$

defines a Marcinkiewicz multiplier on $\mathbb{R} \times \mathbb{R}^{2}$ adapted to the dilations (1.1) on $\mathbb{R}^{2}$. As a consequence, we will obtain that $T_{1}$ is bounded on $L^{p}\left(\mathbb{R}^{3}\right)$. This is part of the folklore, for a formal proof see [13].

It suffices to show that $\widehat{K_{1}}\left(\xi_{1}, \xi\right)$ is a bounded function on $\mathbb{R}^{3}$ such that for each $s_{1} \in\{0,1\}$ and for each multi-index $s=\left(s_{2}, s_{3}\right) \in \mathbb{N}^{2}$ with $|s| \leq 2$ there is a positive constant $C_{s_{1}, s}$ for which

$$
\left|\partial_{\xi_{1}}^{s_{1}} \partial_{\xi}^{s} \widehat{K_{1}}\left(\xi_{1}, \xi\right)\right| \leq C_{s_{1}, s}\left|\xi_{1}\right|^{-s_{1}} \rho(\xi)^{-\frac{s_{2}}{2 n}-\frac{s_{3}}{2 m}}
$$

for every $\left(\xi_{1}, \xi\right) \in \mathbb{R} \times \mathbb{R}^{2}$ with $\xi_{1} \neq 0$ and $\xi \neq 0$. 
We already proved in Proposition 4.1 that $\widehat{K}_{1}\left(\xi_{1}, \xi\right)$ is a bounded function on $\mathbb{R}^{3}$.

We give the proof of the differential inequalities (5.2) for $s_{1}=1$ and $s=(0,0)$ and for $s_{1}=0$ and $s=(1,0)$, the other cases being essentially the same, with the extra disadvantages of more complicated notation and computations.

Set

$$
I_{J}^{\alpha, \beta}\left(\xi_{1}, \xi, \eta\right):=\int_{\frac{1}{2} \leq\left|x_{1}\right| \leq 4} x_{1}^{\alpha}\left(\mathcal{F}_{2}\left(x^{\beta} \varphi_{J}\right)\right)\left(x_{1}, \eta\right) e^{-i\left(\xi_{1}, \xi\right) \cdot\left(x_{1}, \gamma\left(x_{1}\right)\right)} d x_{1},
$$

where $\alpha \in \mathbb{N}, \alpha \leq 2$ and $\beta=\left(\beta_{1}, \beta_{2}\right) \in \mathbb{N}^{2}$ with $|\beta| \leq 2$.

We first consider the case $s_{1}=1$ and $s=(0,0)$. Since

$$
\partial_{\xi_{1}} m_{J}\left(\xi_{1}, \xi\right)=-i 2^{j_{1}} I_{J}^{1,(0,0)}\left(2^{j_{1}} \xi_{1}, 2^{2 m n j_{1}} \circ \xi, 2^{j} \circ \xi\right),
$$

we write

$$
\begin{aligned}
& \sum_{2 m n j_{1} \leq j}\left|\partial_{\xi_{1}} m_{J}\left(\xi_{1}, \xi\right)\right| \leq \\
& \leq\left|\xi_{1}\right|^{-1}\left(\sum_{\substack{2^{j_{1}}\left|\left\|\left(\xi_{1}, \xi\right)\right\|\right| \leq 1 \\
j \in \mathbb{Z}}} 2^{j_{1}}\left|\xi_{1}\right|\left|I_{J}^{1,(0,0)}\left(2^{j_{1}} \xi_{1}, 2^{2 m n j_{1}} \circ \xi, 2^{j} \circ \xi\right)\right|\right. \\
& +\sum_{\substack{2^{j_{1}}\left\|\left(\mid \xi_{1}, \xi\right)\right\| \|>>1 \\
2 m n j_{1} \leq j \\
\text { and } \\
2^{j_{1}}\left|\xi_{1}\right| \leq \tilde{C}\left(2^{m j_{1}}\left|\xi_{2}\right|+2^{n j_{1}}\left|\xi_{3}\right|\right)}} 2^{j_{1}}\left|\xi_{1}\right|\left|I_{J}^{1,(0,0)}\left(2^{j_{1}} \xi_{1}, 2^{2 m n j_{1}} \circ \xi, 2^{j} \circ \xi\right)\right| \\
& \left.+\sum_{\substack{2^{j_{1}}\|\|\left(\xi_{1}, \xi\right)\|\|\left|>1 \\
2 m n j_{1} \leq j \\
2^{j_{1}}\right| \xi_{1} \mid>\tilde{C}\left(2^{m j_{1}}\left|\xi_{2}\right|+2^{n j_{1}}\left|\xi_{3}\right|\right)}} 2^{j_{1}}\left|\xi_{1}\right|\left|I_{J}^{1,(0,0)}\left(2^{j_{1}} \xi_{1}, 2^{2 m n j_{1}} \circ \xi, 2^{j} \circ \xi\right)\right|\right) \\
& =: \Sigma_{1}+\Sigma_{2}+\Sigma_{3} \text {. }
\end{aligned}
$$

Since $2^{j_{1}}\left|\xi_{1}\right| \leq 2^{j_{1}}||\left|\left(\xi_{1}, \xi\right)\right| \|$, the convergence of $\Sigma_{1}$ follows from the estimate (3.12) applied to the integral $I_{J}^{1,(0,0)}\left(2^{j_{1}} \xi_{1}, 2^{2 m n j_{1}} \circ \xi, 2^{j} \circ \xi\right)$.

The inequalities $2^{j_{1}}\left|\xi_{1}\right| \leq \tilde{C}\left(2^{m j_{1}}\left|\xi_{2}\right|+2^{n j_{1}}\left|\xi_{3}\right|\right)$ and $2 m n j_{1} \leq j$ imply that $2^{j_{1}}\left|\xi_{1}\right| \leq \tilde{C}\left(2^{\frac{j}{2 n}}\left|\xi_{2}\right|+2^{\frac{j}{2 m}}\left|\xi_{3}\right|\right) \leq \tilde{C}\left(\left(2^{j} \rho(\xi)\right)^{\frac{1}{2 n}}+\left(2^{j} \rho(\xi)\right)^{\frac{1}{2 m}}\right)$. This fact, together with the estimate (3.14) for the integral $I_{J}^{1,(0,0)}\left(2^{j_{1}} \xi_{1}, 2^{2 m n j_{1}} \circ \xi, 2^{j} \circ \xi\right)$ shows that also $\Sigma_{2}$ converge to a bounded function. Finally, the sum $\Sigma_{3}$ converges because of (3.21). Therefore (5.2) holds for $s_{1}=1$ and $s=(0,0)$.

Now, assume that $s_{1}=0$ and $s=(1,0)$, then we have that

$$
\begin{aligned}
\partial_{\xi_{2}} m_{J}\left(\xi_{1}, \xi\right)= & -i 2^{j / 2 n} I_{J}^{0,(1,0)}\left(2^{j_{1}} \xi_{1}, 2^{2 m n j_{1}} \circ \xi, 2^{j} \circ \xi\right) \\
& -i 2^{m j_{1}} I_{J}^{m,(0,0)}\left(2^{j_{1}} \xi_{1}, 2^{2 m n j_{1}} \circ \xi, 2^{j} \circ \xi\right) .
\end{aligned}
$$


We write

$$
\begin{aligned}
& \sum_{2 m n j_{1} \leq j}\left|\partial_{\xi_{2}} m_{J}\left(\xi_{1}, \xi\right)\right| \leq \\
& \leq \rho(\xi)^{-\frac{1}{2 n}}\left(\sum_{\substack{2^{j_{1}} \|\left(\mid\left(\xi_{1}, \xi\right)\|\| \leq 1 \\
j \in \mathbb{Z}\right.}}\left(2^{j} \rho(\xi)\right)^{\frac{1}{2 n}}\left|I_{J}^{0,(1,0)}\left(2^{j_{1}} \xi_{1}, 2^{2 m n j_{1}} \circ \xi, 2^{j} \circ \xi\right)\right|\right.
\end{aligned}
$$

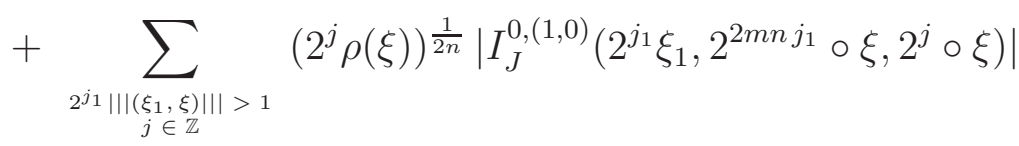

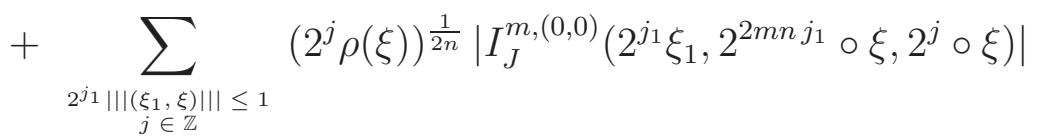

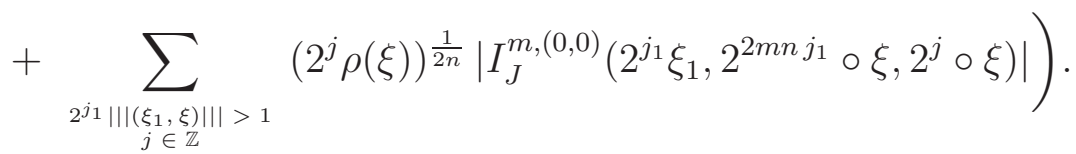

By using the estimate (3.16) we can easily prove that the first and the third series on the right-hand side of (5.4) converge to a bounded function. Also, the second and the fourth series on the right-hand side of (5.4) converge to a bounded function as we can see by applying the estimate (3.14). Hence (5.2) holds for $s_{1}=0$ and $s=(1,0)$.

We now prove the $L^{p}$-boundedness of the operator $T_{2}$ by means of the analytic interpolation method. We start constructing an analytic family of linear operators $T_{2, z}$.

For $z \in \mathbb{C}$ we consider the kernel and its analytic continuation, defined in $(2.9)$,

$$
I^{z}(u)=\frac{G(0) \rho(u)^{z-Q}}{\sigma(S) \Gamma(z) G(z)}
$$

on $\mathbb{R}^{2}$, where $u=\left(u_{2}, u_{3}\right), \sigma(S)$ denotes the surface measure of the sphere

$$
S:=\left\{u \in \mathbb{R}^{2}: \rho(u)=1\right\}
$$

and $G(z)$ has been defined in (2.8).

Example 5.2. In the light of Example 2.4, when $m=2$ and $n=3$ we have

$$
\begin{aligned}
G(z):= & \left(\Gamma\left(z+\frac{7}{12}\right)\right)^{2} \cdot \Gamma\left(z+\frac{1}{6}\right) \Gamma\left(z+\frac{1}{4}\right) \Gamma\left(z+\frac{1}{3}\right) \Gamma\left(z+\frac{5}{12}\right) \\
& \Gamma\left(z+\frac{1}{2}\right) \Gamma\left(z+\frac{2}{3}\right) \Gamma\left(z+\frac{3}{4}\right) \Gamma\left(z+\frac{5}{6}\right) \Gamma\left(z+\frac{11}{12}\right) .
\end{aligned}
$$


We shall denote by $B_{r}, r>0$, the non-isotropic ball

$$
B_{r}:=\left\{\left(u_{1}, u_{2}\right) \in \mathbb{R}^{2}: u_{1}^{2 n}+u_{2}^{2 m} \leq r\right\} .
$$

Let $\theta$ be a smooth compactly supported function on $\mathbb{R}^{2}$ whose support is contained in the ball $B_{\frac{1}{4}}$ and which is identically one in a neighbourhood of the origin. Let $B^{z}$ be the distribution defined by

$$
\left\langle B^{z}, h\right\rangle:=\left\langle I^{z}, \theta h\right\rangle, \quad h \in \mathcal{S}\left(\mathbb{R}^{2}\right) .
$$

Observe that $B^{0}=\delta_{0}$, since $I^{0}=\delta_{0}$ and $\theta$ is identically one in a neighbourhood of the origin.

By considering the convolution between $2^{-j_{1}-j Q} \varphi_{J}\left(2^{-j_{1}} x_{1}, 2^{-j} \circ(x-\right.$ $\left.\gamma\left(x_{1}\right)\right)$ and $2^{-(m+n) j_{1}}\left(\delta_{0} \otimes B^{z}\right)\left(x_{1}, 2^{-2 m n j_{1}} \circ x\right)$ we get the kernel

$$
\begin{aligned}
K_{2, z}\left(x_{1}, x\right)= & \sum_{2 m n j_{1}>j} 2^{-(m+n+1) j_{1}-j Q} \times \\
& \times \int \varphi_{J}\left(2^{-j_{1}} x_{1}, 2^{-j} \circ\left(u-\gamma\left(x_{1}\right)\right)\right) B^{z}\left(2^{-2 m n j_{1}} \circ(x-u)\right) d u .
\end{aligned}
$$

If we set

$$
\lambda_{J}\left(x_{1}, x\right):=2^{\left(2 m n j_{1}-j\right) Q} \int \varphi_{J}\left(x_{1}, 2^{2 m n j_{1}-j} \circ\left(x-u-\gamma\left(x_{1}\right)\right)\right) B^{z}(u) d u
$$

then the kernel $K_{2, z}$ may be written, by a change of variable, as

$$
K_{2, z}\left(x_{1}, x\right):=\sum_{2 m n j_{1}>j} 2^{-(m+n+1) j_{1}} \lambda_{J}\left(2^{-j_{1}} x_{1}, 2^{-2 m n j_{1}} \circ x\right) .
$$

We consider the analytic family of operators (of admissible growth)

$$
T_{2, z} f:=f * K_{2, z}, \quad f \in \mathcal{S}\left(\mathbb{R}^{3}\right),
$$

and we observe that $T_{2,0} f=f * K_{2,0}=T_{2} f$.

In order to prove the $L^{p}$-boundedness of $T_{2}$ we need some preliminary results. The first result is an $L^{1}$ - Lipschitz condition for the distribution $B^{z}$ defined by (5.7).

Lemma 5.3. For all $0<\Re e z<Q$

$$
\int\left|B^{z}(u+h)-B^{z}(u)\right| d u \leq C_{z} \rho(h)^{\Re e z}
$$

for all $h \in \mathbb{R}$. 
Proof. First we split the integral in (5.11) in two parts

$$
\begin{aligned}
\int\left|B^{z}(u+h)-B^{z}(u)\right| d u= & \int_{\rho(h) \leq \frac{\rho(u)}{2}}\left|B^{z}(u+h)-B^{z}(u)\right| d u \\
& +\int_{\rho(h)>\frac{\rho(u)}{2}}\left|B^{z}(u+h)-B^{z}(u)\right| d u=: I+I I .
\end{aligned}
$$

Now

$$
\begin{aligned}
I \leq \int_{\rho(h) \leq \frac{\rho(u)}{2}}\left|C_{z} \theta(u+h)\left(\rho^{z-Q}(u+h)-\rho^{z-Q}(u)\right)\right| d u \\
\quad+\int_{\rho(h) \leq \frac{\rho(u)}{2}}\left|C_{z}(\theta(u+h)-\theta(u)) \rho^{z-Q}(u)\right| d u=: I_{a}+I_{b} .
\end{aligned}
$$

To estimate $I_{a}$, we use the Mean Value Theorem [5, p. 11], obtaining

$$
\begin{aligned}
I_{a} & \leq C \int_{\rho(h) \leq \frac{\rho(u)}{2}}(\rho(u))^{\Re e z-Q-1} \rho(h) d u \\
& =C \rho(h) \int_{2 \rho(h)}^{+\infty} r^{\Re e z-Q-1} r^{Q-1} d r \int_{S} \rho(v)^{\Re e z-Q-1} d \sigma(v) \\
& \leq C \rho(h) \rho(h)^{\Re e z-1}=C \rho(h)^{\Re e z} .
\end{aligned}
$$

To estimate $I_{b}$, we observe that

$$
\begin{aligned}
I_{b} & \leq C \int_{\rho(h) \leq \frac{\rho(u)}{2}}|\theta(u+h)-\theta(u)|(\rho(u))^{\Re e z-Q} d u \\
& \leq C \rho(h)^{\Re e z-Q} \int_{\rho(h) \leq \frac{\rho(u)}{2}}|\theta(u+h)-\theta(u)| d u \leq 2 C \rho(h)^{\Re e z-Q} \int|\theta(u)| d u \\
& \leq C \rho(h)^{\Re e z-Q} .
\end{aligned}
$$

Finally, if $k$ denotes a positive constant such that $\rho(x+y) \leq k(\rho(x)+\rho(y))$ we observe that by $[5, \mathrm{p} .14]$

$$
\begin{aligned}
I I & =\int_{\rho(h)>\frac{\rho(u)}{2}}\left|B^{z}(u+h)-B^{z}(u)\right| d u \leq 2 C \int_{\rho(h)>\frac{\rho(u)}{3 k}}(\rho(u))^{\Re e z-Q} d u \\
& =2 C \int_{0}^{3 k \rho(h)} r^{\Re e z-Q} r^{Q-1} d r \int_{S} \rho(v)^{\Re e z-Q} d \sigma(v) \leq C \rho(h)^{\Re e z} .
\end{aligned}
$$

This inequality, combined with the bounds for $I$, yields (5.11).

Then we need to recall the definition of non-isotropic Besov spaces [15].

Definition 5.4. In $\mathbb{R}^{3}$ we consider the family of one-parameter non-isotropic dilations defined in (3.8). Let $\widetilde{\rho}\left(x_{1}, x\right)$ be any homogeneous norm with respect to these dilations. 
We denote by $B_{1, \infty}^{\alpha}$ the non-isotropic Besov space of functions $f \in L^{1}\left(\mathbb{R}^{3}\right)$ which satisfy an $L^{1}$-Lipschitz condition of order $\alpha, 0<\alpha<1$, i.e. there exists a positive constant $C$ such that

$$
\int\left|f\left(x_{1}+h_{1}, x+h\right)-f\left(x_{1}, x\right)\right| d x_{1} d x \leq C \widetilde{\rho}\left(h_{1}, h\right)^{\alpha}
$$

for every $\left(h_{1}, h\right) \in \mathbb{R} \times \mathbb{R}^{2}$, where $h=\left(h_{2}, h_{3}\right)$.

If $f \in B_{1, \infty}^{\alpha}$ we set

$\|f\|_{B_{1, \infty}^{\alpha}}:=\|f\|_{1}+\sup _{\left(h_{1}, h\right) \neq(0,0)} \widetilde{\rho}\left(h_{1}, h\right)^{-\alpha} \int\left|f\left(x_{1}+h_{1}, x+h\right)-f\left(x_{1}, x\right)\right| d x_{1} d x$.

Our proof hinges on the following result.

Theorem 5.5. Let $\left\{\psi_{l}\right\}_{l \in \mathbb{Z}}$ be a family of functions such that for some positive constants $C, \alpha, \varepsilon$ the following hypotheses hold uniformly in l:

(i) $\left\{\psi_{l}\right\}_{l \in \mathbb{Z}} \subset L^{1}\left(\mathbb{R}^{3}\right)$;

(ii) $\int\left|\psi_{l}\left(x_{1}, x\right)\right|\left(1+\widetilde{\rho}\left(x_{1}, x\right)\right)^{\varepsilon} d x_{1} d x \leq C$;

(iii) $\int \psi_{l}\left(x_{1}, x\right) d x_{1} d x=0$;

(iv) $\left\|\psi_{l}\right\|_{B_{1, \infty}^{\alpha}} \leq C$.

Then the series $\sum_{l \in \mathbb{Z}} 2^{-(m+n+1) l} \psi_{l}\left(2^{-l} \bullet \cdot\right)$ converges in the sense of distributions to a Calderón-Zygmund kernel.

Finally we can state and prove the $L^{p}$-bounds for $T_{2}$.

Proposition 5.6. The operator $T_{2}$ is bounded on $L^{p}\left(\mathbb{R}^{3}\right)$ for $1<p<\infty$.

Proof. The proof is by complex interpolation. We first prove that $T_{2, z}$ is bounded on $L^{2}\left(\mathbb{R}^{3}\right)$ for $-\frac{1}{2 m n^{2}}<\Re e z<0$.

En easy computation shows that

$$
\begin{aligned}
\left(2^{-(m+n+1) j_{1}} \lambda_{J}\left(2^{-j_{1}} \cdot,\left(2^{-2 m n j_{1}} \circ \cdot\right)\right)^{\wedge}\left(\xi_{1}, \xi\right)\right. & =\widehat{\lambda_{J}}\left(2^{j_{1}} \xi_{1}, 2^{2 m n j_{1}} \circ \xi\right) \\
& =\widehat{B^{z}}\left(2^{2 m n j_{1}} \circ \xi\right) m_{J}\left(\xi_{1}, \xi\right),
\end{aligned}
$$

where $m_{J}$ is the Fourier transform of $2^{-j_{1}-j Q} \varphi_{J}\left(2^{-j_{1}} x_{1}, 2^{-j} \circ\left(x-\gamma\left(x_{1}\right)\right)\right.$ and it is given by (4.2), $\lambda_{J}$ is defined by (5.8), and $\widehat{B^{z}}$ is the Fourier transform of $2^{-(m+n) j_{1}}\left(\delta_{0} \otimes B^{z}\left(\xi_{1}, 2^{-2 m n j_{1}} \circ x\right)\right)$. 
It follows from Proposition 2.8 that

$$
\left|\widehat{B^{z}}(\xi)\right| \leq C(1+\rho(\xi))^{-\Re e z} .
$$

Our aim is now to prove that the series

$$
\sum_{2 m n j_{1}>j} \widehat{B^{z}}\left(2^{2 m n j_{1}} \circ \xi\right) m_{J}\left(\xi_{1}, \xi\right)
$$

corresponding to the Fourier transform of (5.9), converges boundedly to a bounded function. The inequality (5.12) yields

$$
\begin{aligned}
\left|\sum_{2 m n j_{1}>j} \widehat{B^{z}}\left(2^{2 m n j_{1}} \circ \xi\right) m_{J}\left(\xi_{1}, \xi\right)\right| \leq \\
\leq C_{z} \sum_{\substack{2^{j_{1}} \mid\left\|\left(\xi_{1}, \xi\right)\right\| \| \leq 1 \\
2 m n j_{1}>j}}\left(1+\rho\left(2^{2 m n j_{1}} \circ \xi\right)\right)^{|\Re e z|}\left|m_{J}\left(\xi_{1}, \xi\right)\right| \\
\quad+\sum_{\substack{2^{j_{1}} \mid\left\|\left(\xi_{1}, \xi\right)\right\| \|>1 \\
2 m n j_{1}>j}}\left(1+\rho\left(2^{2 m n j_{1}} \circ \xi\right)\right)^{|\Re e z|}\left|m_{J}\left(\xi_{1}, \xi\right)\right|=: \mathrm{J}_{1}+\mathrm{J}_{2} .
\end{aligned}
$$

Since

$$
2^{2 m n j_{1}} \rho(\xi)=2^{2 m n j_{1}}\left(\xi_{2}^{2 n}+\xi_{3}^{2 m}\right) \leq 2 \cdot\left(2^{j_{1}}||\left|\left(\xi_{1}, \xi\right)\right|||\right)^{2 m n},
$$

we have

$$
\mathrm{J}_{1} \leq C 3^{|\Re e z|} \sum_{\substack{2^{j_{1}}\left\|\left(\xi_{1}, \xi\right)\right\| \mid \leq 1 \\ 2 m n j_{1}>j}}\left|m_{J}\left(\xi_{1}, \xi\right)\right| \leq C_{z},
$$

In the light of what has been proved in Proposition 4.1, to estimate $\mathrm{J}_{2}$ observe that

$$
\mathrm{J}_{2} \leq C_{z} \sum_{\substack{2^{j_{1}}\left\|\left(\mid \xi_{1}, \xi\right)\right\| \|>1 \\ 2 m n \\ j_{1}>j}}\left(2^{j_{1}}||\left|\left(\xi_{1}, \xi\right)\right|||\right)^{2 m n|\Re e z|}\left|m_{J}\left(\xi_{1}, \xi\right)\right| .
$$

Now, by using (4.6) and estimate (3.14) for $I_{J}$, we obtain

$$
\mathrm{J}_{2} \leq C_{z} \sum_{\substack{2^{j_{1}}\left\|\mid\left(\xi_{1}, \xi\right)\right\| \|>1 \\ 2 m n j_{1}>j}} \frac{\left(2^{j_{1}}||\left|\left(\xi_{1}, \xi\right)\right|||\right)^{2 m n|\Re e z|}}{\left(2^{j_{1}}||\left|\left(\xi_{1}, \xi\right)\right| \|\right)^{1 / n}} \frac{\rho\left(2^{j} \circ \xi\right)^{1 / 2 n}}{\left(1+\rho\left(2^{j} \circ \xi\right)\right)^{N}},
$$

so that the operator $T_{2, z}$ is bounded on $L^{2}\left(\mathbb{R}^{3}\right)$ if $-\frac{1}{2 m n^{2}}<\Re e z<0$.

We will now show that the operator $T_{2, z}$ is bounded on $L^{p}\left(\mathbb{R}^{3}\right)$ for $1<$ $p<\infty$ for $0<\Re e z<Q$. 
By setting $j-2 m n j_{1}=k$ and using (3.10), (5.9) may be written as

$$
K_{2, z}\left(x_{1}, x\right)=\sum_{j_{1} \in \mathbb{Z}} 2^{-(m+n+1) j_{1}} \sum_{k=-\infty}^{0} \lambda_{\left(j_{1}, k+2 m n j_{1}\right)}\left(2^{-j_{1}} \bullet\left(x_{1}, x\right)\right)
$$

where

$$
\begin{aligned}
& \lambda_{\left(j_{1}, k+2 m n j_{1}\right)}\left(x_{1}, x\right)= \\
& \quad=2^{-Q k} \int \varphi_{\left(j_{1}, k+2 m n j_{1}\right)}\left(x_{1}, 2^{-k} \circ\left(x-u-\gamma\left(x_{1}\right)\right)\right) B^{z}(u) d u .
\end{aligned}
$$

We shall now prove that the functions

$$
\psi_{j_{1}}\left(x_{1}, x\right):=\sum_{k=-\infty}^{0} \lambda_{\left(j_{1}, k+2 m n j_{1}\right)}\left(x_{1}, x\right)
$$

satisfy the hypotheses of Theorem 5.5 uniformly in $j_{1}$. Since the estimates on the functions $\psi_{j_{1}}\left(x_{1}, x\right)$ that we will prove later are independent of $j_{1}$, it suffices to prove that

$$
\psi_{0}\left(x_{1}, x\right)=\sum_{k=-\infty}^{0} \lambda_{(0, k)}\left(x_{1}, x\right)
$$

satisfies the hypotheses of Theorem 5.5.

We begin proving that $\psi_{0}$ belongs to $L^{1}\left(\mathbb{R}^{3}\right)$. As a consequence of (3.5) with $\beta=0$ and a change of variable we obtain

$\psi_{0}\left(x_{1}, x\right)=\sum_{k=-\infty}^{0} \int \varphi_{(0, k)}\left(x_{1}, v\right)\left(B^{z}\left(x-2^{k} \circ v-\gamma\left(x_{1}\right)\right)-B^{z}\left(x-\gamma\left(x_{1}\right)\right)\right) d v$,

whence

$$
\begin{aligned}
& \int\left|\psi_{0}\left(x_{1}, x\right)\right| d x_{1} d x \leq \\
& \leq \sum_{k=-\infty}^{0} \int\left(\int\left|\varphi_{(0, k)}\left(x_{1}, v\right)\right| \cdot\left|B^{z}\left(x-2^{k} \circ v-\gamma\left(x_{1}\right)\right)-B^{z}\left(x-\gamma\left(x_{1}\right)\right)\right| d v\right) d x_{1} d x \\
& =\sum_{k=-\infty}^{0} \int\left|\varphi_{(0, k)}\left(x_{1}, v\right)\right|\left(\int\left|B^{z}\left(x-2^{k} \circ v\right)-B^{z}(x)\right| d x\right) d x_{1} d v \\
& \leq C_{z} \sum_{k=-\infty}^{0} \int\left|\varphi_{(0, k)}\left(x_{1}, v\right)\right| \rho\left(2^{k} \circ v\right)^{\Re e z} d x_{1} d v \\
& =C_{z} \sum_{k=-\infty}^{0} 2^{k \Re e z} \int\left|\varphi_{(0, k)}\left(x_{1}, v\right)\right| \rho(v)^{\Re e z} d x_{1} d v \leq C_{z},
\end{aligned}
$$

by Lemma 5.3 and since $\left\{\varphi_{(0, k)}\right\}$ is a bounded set in $\mathcal{S}\left(\mathbb{R}^{3}\right)$. 
Now we shall prove that $\psi_{0}$ satisfies the hypothesis $i i$ ) of Theorem 5.5 with $\varepsilon=1$ and

$$
\widetilde{\rho}\left(x_{1}, x\right)=\left|x_{1}\right|+\left|x_{2}\right|^{\frac{1}{m}}+\left|x_{3}\right|^{\frac{1}{n}} .
$$

We split the integral as

$$
\begin{aligned}
& \int\left|\psi_{0}\left(x_{1}, x\right)\right|\left(1+\widetilde{\rho}\left(x_{1}, x\right)\right) d x_{1} d x \\
& \quad=\left(\int_{\substack{\frac{1}{2} \leq\left|x_{1}\right| \leq 4 \\
\left|x_{2}\right| \frac{1}{m}+\left|x_{3}\right|^{\frac{1}{n}} \leq M}}+\int_{\substack{\frac{1}{2} \leq\left|x_{1}\right| \leq 4 \\
\left|x_{2}\right|^{\frac{1}{m}}+\left|x_{3}\right|^{\frac{1}{n}}>M}}\right)\left|\psi_{0}\left(x_{1}, x\right)\right|\left(1+\widetilde{\rho}\left(x_{1}, x\right)\right) d x_{1} d x \\
& =\Im_{1}+\Im_{2},
\end{aligned}
$$

where the costant $M$ will be chosen later.

Now $\Im_{1}$ is bounded by some positive costant $C$, since $\psi_{0}\left(x_{1}, x\right)$ belongs to $L^{1}\left(\mathbb{R}^{3}\right)$ and $\widetilde{\rho}\left(x_{1}, x\right)$ is bounded on the integration set. To study $\Im_{2}$, observe that

$$
\begin{aligned}
& \Im_{2} \leq \sum_{k=-\infty}^{0} 2^{-k Q} \int_{\substack{\frac{1}{2} \leq\left|x_{1}\right| \leq 4 \\
\left|x_{2}\right|^{\frac{1}{m}}+\left|x_{3}\right|^{\frac{1}{n}}>M}} \int_{\rho(u) \leq \frac{1}{4}} \mid \varphi_{(0, k)}\left(x_{1}, 2^{-k} \circ\left(x-u-\gamma\left(x_{1}\right)\right) \mid \times\right. \\
& \times\left|B^{z}(u)\right| d u\left(5+\left|x_{2}\right|^{\frac{1}{m}}+\left|x_{3}\right|^{\frac{1}{n}}\right) d x_{1} d x \\
& \leq C \sum_{k=-\infty}^{0} 2^{-k Q} \int_{\substack{\frac{1}{2} \leq\left|x_{1}\right| \leq 4 \\
\left|x_{2}\right|^{\frac{1}{m}}+\left|x_{3}\right|^{\frac{1}{n}}>M}} \int_{\rho(u) \leq \frac{1}{4}} \frac{|| \varphi_{(0, k)}||_{(N)}\left|B^{z}(u)\right|}{\left(1+\left|2^{-k} \circ\left(x-u-\gamma\left(x_{1}\right)\right)\right|\right)^{N}} d u \times \\
& \times\left(5+\left|x_{2}\right|^{\frac{1}{m}}+\left|x_{3}\right|^{\frac{1}{n}}\right) d x_{1} d x \\
& \leq C \sum_{k=-\infty}^{0} 2^{-k Q} \int_{\substack{\frac{1}{2} \leq\left|x_{1}\right| \leq 4 \\
\left|x_{2}\right|^{\frac{1}{m}}+\left|x_{3}\right|^{\frac{1}{n}}>M}}\left(5+\left|x_{2}\right|^{\frac{1}{m}}+\left|x_{3}\right|^{\frac{1}{n}}\right) \times \\
& \times \int_{\rho(u) \leq \frac{1}{4}} \frac{|| \varphi_{(0, k)}||_{(N)}\left|B^{z}(u)\right|}{\left(1+\left|2^{-k} \circ x\right|\right)^{N}} d u d x_{1} d x,
\end{aligned}
$$

where the last inequality follows from the fact that for some constant $C>0$ we have

$$
1+\left|2^{-k} \circ\left(x-u-\gamma\left(x_{1}\right)\right)\right| \geq C\left(1+\left|2^{-k} \circ x\right|\right)
$$

if $\left|x_{2}\right|^{\frac{1}{m}}+\left|x_{3}\right|^{\frac{1}{n}}>M$, if $M$ is sufficiently large, $\rho(u) \leq \frac{1}{4}, \frac{1}{2} \leq\left|x_{1}\right| \leq 4$.

Next (2.2) yields

$$
\left|2^{-k} \circ x\right| \geq A\left(\rho\left(2^{-k} \circ x\right)\right)^{\frac{1}{2 n}}
$$

for some $A>0$, if $k \leq 0$ and $\left|x_{2}\right|^{\frac{1}{m}}+\left|x_{3}\right|^{\frac{1}{n}}>M$. 
Now (5.16) and the local integrability of $B^{z}$ give

$$
\begin{aligned}
\Im_{2} & \leq C \sum_{k=-\infty}^{0} 2^{-k Q} \int_{\left|x_{2}\right|^{\frac{1}{m}}+\left|x_{3}\right|^{\frac{1}{n}}>M}\left(5+\left|x_{2}\right|^{\frac{1}{m}}+\left|x_{3}\right|^{\frac{1}{n}}\right) \frac{1}{\left(1+\rho\left(2^{-k} \circ x\right)\right)^{\frac{N}{2 n}}} d x \\
& \leq C \sum_{k=-\infty}^{0} 2^{-k Q} \int_{\left|x_{2}\right|^{\frac{1}{m}}+\left|x_{3}\right|^{\frac{1}{n}}>M}\left(5+\left|x_{2}\right|^{\frac{1}{m}}+\left|x_{3}\right|^{\frac{1}{n}}\right) \frac{1}{\left(1+2^{-k} \rho(x)\right)^{\frac{N}{2 n}}} d x \\
& \leq C \sum_{k=-\infty}^{0} 2^{-k Q_{2} 2^{\frac{k N}{2 n}}} \int_{\left|x_{2}\right|^{\frac{1}{m}}+\left|x_{3}\right|^{\frac{1}{n}}>M}\left(5+\left|x_{2}\right|^{\frac{1}{m}}+\left|x_{3}\right|^{\frac{1}{n}}\right) \frac{1}{\rho(x)^{\frac{N}{2 n}}} d x \leq C,
\end{aligned}
$$

yielding $i i)$.

Property iii) is an immediate consequence of (3.5).

Finally we shall prove that $\psi_{0}$ satisfies property $i v$ ) in Theorem 5.5.

We start proving that

$$
\int\left|\psi_{0}\left(x_{1}, x+h\right)-\psi_{0}\left(x_{1}, x\right)\right| d x_{1} d x \leq C_{z}^{\prime} \rho(h)^{\frac{\Re e z}{4 m n}}, h \in \mathbb{R}^{2} .
$$

Indeed we have

$$
\begin{aligned}
& \int\left|\psi_{0}\left(x_{1}, x+h\right)-\psi_{0}\left(x_{1}, x\right)\right| d x_{1} d x \leq \\
& \leq \sum_{k=-\infty}^{0} \int\left(\int \mid \varphi_{(0, k)}\left(x_{1}, v\right)\left(B^{z}\left(x+h-2^{k} \circ v-\gamma\left(x_{1}\right)\right)-B^{z}\left(x+h-\gamma\left(x_{1}\right)\right)\right)\right. \\
& \left.\quad-\varphi_{(0, k)}\left(x_{1}, v\right)\left(B^{z}\left(x-2^{k} \circ v-\gamma\left(x_{1}\right)\right)-B^{z}\left(x-\gamma\left(x_{1}\right)\right)\right) \mid d x d x_{1}\right) d v \\
& =: \sum_{k=-\infty}^{0} \int J_{k}(v) d v .
\end{aligned}
$$

Now observe that

$$
\begin{aligned}
J_{k}(v) & \leq 2 \int\left|\varphi_{(0, k)}\left(x_{1}, v\right)\left(B^{z}\left(x-2^{k} \circ v-\gamma\left(x_{1}\right)\right)-B^{z}\left(x-\gamma\left(x_{1}\right)\right)\right)\right| d x d x_{1} \\
& =2 \int\left|\varphi_{(0, k)}\left(x_{1}, v\right)\right|\left(\int\left|B^{z}\left(y-2^{k} \circ v\right)-B^{z}(y)\right| d y\right) d x_{1}
\end{aligned}
$$

$$
\leq C_{z, N} \frac{\rho\left(2^{k} \circ v\right)^{\Re e z}}{(1+|v|)^{N}}=C_{z, N} 2^{k \Re e z} \frac{\rho(v)^{\Re e z}}{(1+|v|)^{N}}, \quad N \in \mathbb{N},
$$

where we used both the Lemma 5.3 and the fact that the functions $\varphi_{(0, k)}$ are a bounded set in $\mathcal{S}\left(\mathbb{R}^{3}\right)$. 
On the other hand, as a consequence of Lemma 5.3

$J_{k}(v) \leq 2 \int\left|\varphi_{(0, k)}\left(x_{1}, v\right) B^{z}\left(y+h-\gamma\left(x_{1}\right)\right)-\varphi_{(0, k)}\left(x_{1}, v\right) B^{z}\left(y-\gamma\left(x_{1}\right)\right)\right| d y d x_{1}$

$$
\leq C_{z, N} \frac{\rho(h)^{\Re e z}}{(1+|v|)^{N}}, \quad N \in \mathbb{N}, h \in \mathbb{R}^{2} .
$$

By taking a suitable mean between estimates (5.18) and (5.19) we obtain

$$
J_{k}(v)=J_{k}(v)^{\frac{4 m n-1}{4 m n}} \cdot J_{k}(v)^{\frac{1}{4 m n}} \leq C_{z, N} 2^{\frac{4 m n-1}{4 m n} k \Re e z} \rho(h)^{\frac{\Re e z}{4 m n}} \frac{\rho(v)^{\frac{4 m n-1}{4 m n} \Re e z}}{(1+|v|)^{N}},
$$

for $N \in \mathbb{N}, h \in \mathbb{R}$, so that

$$
\begin{aligned}
& \int\left|\psi_{0}\left(x_{1}, x+h\right)-\psi_{0}\left(x_{1}, x\right)\right| d x_{1} d x \leq \sum_{k=-\infty}^{0} \int J_{k}(v) d v \\
& \leq C_{z, N} \sum_{k=-\infty}^{0} 2^{\frac{4 m n-1}{4 m n} k \Re e z} \rho(h)^{\frac{\Re e z}{4 m n}} \int \frac{\rho(v)^{\frac{4 m n-1}{4 m n} \Re e z}}{(1+|v|)^{N}} d v \leq C_{z, N} \rho(h)^{\frac{\Re e z}{4 m n}},
\end{aligned}
$$

proving (5.17).

In an analogous way we can prove the inequality

$$
\int\left|\psi_{0}\left(x_{1}+h_{1}, x\right)-\psi_{0}\left(x_{1}, x\right)\right| d x_{1} d x \leq C_{z}^{\prime}\left|h_{1}\right|^{\frac{\Re e z}{2}} .
$$

Finally we have

$$
\begin{gathered}
\int\left|\psi_{0}\left(x_{1}+h_{1}, x+h\right)-\psi_{0}\left(x_{1}, x\right)\right| d x_{1} d x \leq \\
\leq \int\left|\psi_{0}\left(x_{1}+h_{1}, x+h\right)-\psi_{0}\left(x_{1}+h_{1}, x\right)\right| d x_{1} d x \\
\quad+\int\left|\psi_{0}\left(x_{1}+h_{1}, x\right)-\psi_{0}\left(x_{1}, x\right)\right| d x_{1} d x \\
\leq C_{z}\left(\left|h_{1}\right|^{\frac{\Re e z}{2}}+\rho(h)^{\frac{\Re e z}{4 m n}}\right)
\end{gathered}
$$

Since

$$
\left|h_{1}\right|^{\frac{\Re e z}{2}}+\rho(h)^{\frac{\Re e z}{4 m n}} \leq C\left(\left|h_{1}\right|+\rho(h)^{\frac{1}{2 m n}}\right)^{\frac{\Re e z}{2}}
$$

and, by a standard inequality,

$$
\left(h_{2}^{2 n}+h_{3}^{2 m}\right)^{\frac{1}{2 m n}} \leq\left|h_{2}\right|^{\frac{1}{m}}+\left|h_{3}\right|^{\frac{1}{n}},
$$

we finally get

$$
\left|h_{1}\right|^{\frac{\Re e z}{2}}+\rho(h)^{\frac{\Re e z}{4 m n}} \leq C \tilde{\rho}\left(h_{1}, h\right)^{\frac{\Re e z}{2}},
$$

proving (iv) with $\alpha=\Re e z / 2$. As a consequence of Theorem 5.5 the operator $T_{2, z}$ is bounded on $L^{p}\left(\mathbb{R}^{3}\right), 1<p<\infty$, for $0<\Re e z<Q$. 
Finally choose $p_{0} \in(1,2)$ and fix $q_{0} \in\left(1, p_{0}\right)$ such that

$$
\frac{1}{p_{0}}=\frac{b}{2(b-a)}-\frac{a}{q_{0}(b-a)} \text {. }
$$

Then the operator $T_{2,0}=T_{2}$ is bounded on $L^{p_{0}}\left(\mathbb{R}^{3}\right)$. By the arbitrariness of $p_{0}$ and by duality we conclude that $T_{2,0}=T_{2}$ is bounded on $L^{p}\left(\mathbb{R}^{3}\right)$ for all $1<p<\infty$.

\section{Final remarks}

\section{Remark 1}

We observe that our results also hold in the more general situation in which the curve $\gamma\left(x_{1}\right)=\left(x_{1}^{m}, x_{1}^{n}\right)$ is perturbed to $\hat{\gamma}\left(x_{1}\right)=\left(x_{1}^{m}+\lambda_{2}\left(x_{1}\right), x_{1}^{n}+\lambda_{3}\left(x_{1}\right)\right)$ with $\lambda_{2}$ and $\lambda_{3}$ smooth and satisfying $\lambda_{2}\left(x_{1}\right)=o\left(x_{1}^{m}\right)$ and $\lambda_{3}\left(x_{1}\right)=o\left(x_{1}^{n}\right)$. In fact, given a product kernel $K_{0}$ on $\mathbb{R}^{3}$, we define the distribution $K$ by

$$
\int K\left(x_{1}, x\right) f\left(x_{1}, x\right) d x_{1} d x:=\int K_{0}\left(x_{1}, x\right) f\left(x_{1}, x+\hat{\gamma}\left(x_{1}\right)\right) d x_{1} d x
$$

for all Schwartz functions $f$ on $\mathbb{R}^{3}$. Then we break the integral on the right-hand side as

$$
\left(\int_{\mathbb{R}} \int_{-1}^{1}+\int_{\mathbb{R}} \int_{\left|x_{1}\right|>1}\right) K_{0}\left(x_{1}, x\right) f\left(x_{1}, x+\hat{\gamma}\left(x_{1}\right)\right) d x_{1} d x .
$$

The first term, by means of a Taylor expansion, may be reduced to the polynomial case, while the latter one is of Calderon-Zygmund type.

\section{Remark 2}

Our results still hold for convolution operators on $\mathbb{R}^{d}=\mathbb{R} \times \mathbb{R}^{d-1}$ with kernels adapted to curves of the form $\gamma\left(x_{1}\right)=\left(x_{1}^{m_{1}}, x_{1}^{m_{2}}, \ldots, x_{1}^{m_{d-1}}\right), 1<m_{1}<$ $m_{2}<\cdots<m_{d-1}, m_{j} \in \mathbb{N}, j=1, \ldots, d-1$, with values in $\mathbb{R}^{d-1}$. Since notation in the higher dimensional case is more cumbersome, we gave full details of the proof only for the space $\mathbb{R}^{3}$.

On $\mathbb{R}^{d-1}$ we introduce the dilations given by

$$
\delta \circ x=\left(\delta^{1 / 2 m_{d-1}} x_{2}, \delta^{1 / 2 m_{d-2}} x_{3}, \ldots, \delta^{1 / 2 m_{1}}\right),
$$

where $\delta>0$ and $x=\left(x_{2}, \ldots, x_{d-1}\right)$. Moreover we equip $\mathbb{R}^{d-1}$ with the smooth homogeneous norm

$$
\rho(x)=x_{2}^{2 m_{d-1}}+x_{3}^{2 m_{d-2}}+\cdots+x_{d}^{2 m_{1}}
$$

on $\mathbb{R}^{d-1}$. From this point on, the proof follows the same pattern as in $\mathbb{R}^{3}$. 
In particular, the strategy of using Bernstein-Sato polynomials to build the meromorphic continuation of the non isotropic Riesz potentials $\rho(x)^{z-\tilde{Q}}$ (here $\tilde{Q}=\frac{1}{2 m_{d-1}}+\frac{1}{2 m_{d-2}}+\ldots+\frac{1}{2 m_{1}}$ ) works in the multidimensional case as well, with some additional computational difficulties in finding the zeros of the Bernstein-Sato polynomials [1].

\section{Remark 3}

As an example of the class of operators studied in this paper we exhibit the following operator, arising in the study of the $L^{p}-L^{q}$ boundedness of a double analytic family of fractional integrals along curves in the space (see [3] for the planar case).

Let $\psi$ be a smooth function on $\mathbb{R}^{2}$, such that $\psi\left(u_{1},-u_{2}\right)=\psi\left(u_{1}, u_{2}\right)$ for every $\left(u_{1}, u_{2}\right) \in \mathbb{R}^{2}, \psi \equiv 1$ on $B_{\frac{1}{2}}$ and $\psi \equiv 0$ outside $B_{1}$, with $0 \leq \psi \leq 1$ on $\mathbb{R}^{2}$ (here $B_{r}, r>0$, denotes the non-isotropic ball in $\mathbb{R}^{2}$ defined by (5.6)).

Define an analytic family of distributions $K_{z}^{\gamma}$, for $\gamma$ and $z$ in $\mathbb{C}, \Re e \gamma \geq 0$, in the following way

$$
<K_{z}^{\gamma}, f>:=\int<D_{z}\left(u_{1}, u_{2}\right), f\left(t, u_{1} t^{m}, u_{2} t^{n}\right)>|t|^{\gamma} \frac{d t}{t},
$$

where $D_{z}, \Re e z>0$, denotes the family of analytic distributions given by

$$
\begin{aligned}
<D_{z}, h> & :=<\psi(\cdot, \cdot) I^{z}(\cdot, \cdot), h(\cdot+1, \cdot+1)> \\
& =C_{z} \int_{\mathbb{R}^{2}} \rho\left(u_{1}-1, u_{2}-1\right)^{z-Q} \psi\left(u_{1}-1, u_{2}-1\right) h\left(u_{1}, u_{2}\right) d u_{1}, d u_{2},
\end{aligned}
$$

with $C_{z}:=\frac{G(0)}{2 \sigma(S) \Gamma(z) G(z)}$ and $h \in \mathcal{C}_{c}^{\infty}\left(\mathbb{R}^{2}\right)$. It is straightforward to check that $D_{z}$ may be extended to all $z \in \mathbb{C}$.

As a consequence of Proposition 2.6 we have

$$
<D_{0}, h>=h(1,1) .
$$

We remark that, if $\Re e \gamma=0$, then

$$
<K_{z}^{\gamma}, f>:=\lim _{\varepsilon \rightarrow 0} \int<D_{z}\left(u_{1}, u_{2}\right), f\left(t, u_{1} t^{m}, u_{2} t^{n}\right)>|t|^{i \rho+\varepsilon} \frac{d t}{t},
$$

where $\Im m \gamma=\rho$, for every $f \in \mathcal{C}_{c}^{\infty}\left(\mathbb{R}^{2}\right)$. Observe moreover that $K_{z}^{\gamma}$ depends analytically on both $\gamma$ and $z$.

At this point we may introduce the family of convolution operators with kernel $K_{z}^{\gamma}$ defined by (6.2), that is

$$
\begin{aligned}
\left(S_{z}^{\gamma} f\right)\left(x_{1}, x_{2}, x_{3}\right): & =\left(K_{z}^{\gamma} * f\right)\left(x_{1}, x_{2}, x_{3}\right) \\
& =\int<D_{z}\left(u_{1}, u_{2}\right), f\left(x_{1}-t, x_{2}-u_{1} t^{m}, x_{3}-u_{2} t^{n}\right)>|t|^{\gamma} \frac{d t}{t} .
\end{aligned}
$$


We observe that, in the light of (6.3), we have

$$
\left(S_{0}^{\gamma} f\right)\left(x_{1}, x_{2}, x_{3}\right):=C_{0} \int_{\mathbb{R}^{3}} f\left(x_{1}-t, x_{2}-t^{m}, x_{3}-t^{n}\right)>|t|^{\gamma} \frac{d t}{t},
$$

that is, at the height $z=0$ we recover the fractional integration operator along the curve $t \mapsto\left(t, t^{m}, t^{n}\right)$ in the space.

In a forthcoming paper [2] we shall give a complete picture of the characteristic set of the operator $S_{z}^{\gamma}$. A key step in the proof of that result is the fact that at the height $\Re e z=0$ and for $\Re e \gamma=0$ the kernel $K_{z}^{\gamma}$ is a product kernel adapted to the curve $x_{1} \mapsto\left(x_{1}^{m}, x_{2}^{n}\right)$. Thus Theorem 1.2 yields $L^{p}$-boundedness for the operator $S_{z}^{\gamma}$ for $\Re e z=0$ and $\Re e \gamma=0$ for all $p \in(1, \infty)$.

\section{References}

[1] Budur, N., Mustatǎ, M. and Saito, M.: Combinatorial description of the roots of the Bernstein-Sato polynomials for monomial ideals. Comm. Algebra 34 (2006), no. 11, 4103-4117.

[2] Casarino, V., Ciatti, P. And Secco, S.: Product structures and fractional integration along curves in the space. Submitted, 2010.

[3] Casarino, V. and Secco, S.: $L^{p}-L^{q}$ boundedness of analytic families of fractional integrals. Studia Math. 184 (2008), no. 2, 153-174.

[4] Fefferman, R. and Stein, E. M.: Singular integrals on product spaces. Adv. in Math. 45 (1982), no. 2, 117-143.

[5] Folland, G. B. And Stein, E. M.: Hardy Spaces on Homogeneous Groups. Mathematical Notes 28. Princeton University Press, Princeton, NJ, 1982.

[6] Journé, J. L.: Calderón-Zygmund operators on product spaces. Rev. Mat. Iberoamericana 1 (1985), no. 3, 55-92.

[7] Kashiwara, M.: B-functions and holonomic systems. Invent. Math. 38 (1976/77), no. 1, 33-53.

[8] Malgrange, B.: Sur les polynômes de I. N. Bernstein. In Séminaire Goulauic-Schwartz 1973-1974: Ėquations aux dérivées partielles et analyse fonctionnelle, Exp. No. 20, 10 pp. Centre de Math., École Polytech., Paris, 1974.

[9] Müller, D., Ricci, F. And Stein, E. M.: Marcinkiewicz multipliers and multi-parameter structure on Heisenberg (-type) groups. I. Invent. Math. 119 (1995), no. 2, 119-233.

[10] Nagel, A. And Stein, E. M.: On the product theory of singular integrals. Rev. Mat. Iberoamericana 20 (2004), no. 2, 531-561. 
[11] Nagel, A. And Stein, E. M.: The $\bar{\partial}_{b}$-complex on decoupled boundaries in $\mathbb{C}^{n}$. Ann. of Math. (2) 164 (2006), no. 2, 649-713.

[12] Nagel, A., Ricci, F. And Stein, E. M.: Singular integrals with flag kernels and analysis on quadratic CR manifolds. J. Funct. Anal. 181 (2001), no. $1,29-118$.

[13] Ricci, F.: Fourier and spectral multipliers in $\mathbb{R}^{n}$ and the Heisenberg group. Preprint, 2004. Available on line at the following address: http://homepage.sns.it/fricci/papers/multipliers.pdf.

[14] Secco, S.: Adapting product kernels to curves in the plane. Math. Z. 248 (2004), no. 3, 459-476.

[15] Stein, E. M.: Harmonic analysis: real-variable methods, orthogonality and oscillatory integrals. Princeton Mathematical Series 43. Monographs in Harmonic Analysis, III. Princeton University Press, Princeton, NJ, 1993.

[16] Stein, E. M. And Wainger, S.: Problems in harmonic analysis related to curvature. Bull. Amer. Math. Soc. 84 (1978), no. 6, 1239-1295.

Recibido: 5 de noviembre de 2009

Valentina Casarino Dipartimento di Metodi e Modelli Matematici per le Scienze Applicate

Via Trieste 63 I- 35121 Padova, Italy valentina.casarino@unipd.it

Paolo Ciatti

Dipartimento di Metodi e Modelli Matematici per le Scienze Applicate

Via Trieste 63 I-35121 Padova, Italy ciatti@dmsa.unipd.it

Silvia Secco silvia.secco@alice.it

The authors have been partially supported by grant Prin 2007 Analisi Armonica. 